\title{
The effect of coupling conditions on the stability of bimodal systems in $\mathbb{R}^{3}$
}

\author{
Vasfi Eldem ${ }^{\mathrm{a}}$, Gökhan Şahan ${ }^{\mathrm{b}, *}$ \\ ${ }^{a}$ Okan University, Department of Basic Sciences in Engineering, Akfirat-Tuzla İstanbul, Turkey \\ ${ }^{\mathrm{b}}$ Izmir Institute of Technology, Department of Mathematics, Urla, Izmir, Turkey
}

\section{A R T I C L E I N F O}

\section{Article history:}

Received 31 July 2015

Received in revised form

14 March 2016

Accepted 28 July 2016

Available online 7 September 2016

\section{Keywords:}

Bimodal system

Asymptotic stability

Discontinuous vector field

Piecewise linear systems

Coupling condition

\begin{abstract}
A B S T R A C T
This paper investigates the global asymptotic stability of a class of bimodal piecewise linear systems in $\mathbb{R}^{3}$. The approach taken allows the vector field to be discontinuous on the switching plane. In this framework, verifiable necessary and sufficient conditions are proposed for global asymptotic stability of bimodal systems being considered. It is further shown that the way the subsystems are coupled on the switching plane plays a crucial role on global asymptotic stability. Along this line, it is demonstrated that a constant (which is called the coupling constant in the paper) can be changed without changing the eigenvalues of subsystems and this change can make bimodal system stable or unstable.
\end{abstract}

(c) 2016 Elsevier B.V. All rights reserved.

\section{Introduction}

Switched systems consist of a finite number of subsystems and a switching rule that triggers the switching between subsystems. Piecewise linear systems (PLS) comprise a subclass of switched systems where the subsystems are linear and time invariant and the switching rule is autonomous (state dependent). Bimodal piecewise linear systems (BPLS) comprise a subclass of switched linear systems where there are only two subsystems.

Switched systems arise in many engineering and physical problems. Therefore, they have attracted significant attention in the literature. The details of the work done on switched systems can be found in books [1-5] and in the papers [6,7].

One of the main issues in bimodal systems is well-posedness (existence and uniqueness of solutions). This issue is investigated extensively in [8-11]. BPLS is also investigated in the context of stability, stabilizability and control in [12-17,7]. Moreover, BPLS also provide convenient tools to investigate nonlinear phenomenon encountered in biological, physical or engineering processes as demonstrated in $[18,19]$.

BPLS in $\mathbb{R}^{3}$ have also attracted significant attention in the literature. For instance, Carmona et al. [20-22] investigated these systems where the vector field is continuous on the switching

\footnotetext{
* Corresponding author.

E-mail addresses: vasfi.eldem@okan.edu.tr (V. Eldem), gsahan@iyte.edu.tr (G. Şahan).
}

plane. The same problem, without the assumption of continuity of the vector field, is considered in [13,14,23]. Eldem and Sahan [13] have shown that the necessary and sufficient conditions for global asymptotic stability (GAS) in $\mathbb{R}^{3}$ are reduced to the well-known GAS conditions in $\mathbb{R}^{2}$ under an assumption which simplifies the geometric structure of BPLS in $\mathbb{R}^{3}$. Eldem and Öner [14] have investigated the stability of BPLS in $\mathbb{R}^{3}$ in a framework which is similar to ours.

Up to our knowledge a complete set of verifiable necessary and sufficient conditions for GAS of BPLS in $\mathbb{R}^{n}$ and in $\mathbb{R}^{3}$ have not appeared in the literature, yet. Carmona et al., $[20,21]$ have studied the GAS of BPLS in $\mathbb{R}^{3}$ by transforming the system onto the unit sphere centered at the origin. The vector field is assumed to be continuous on the switching plane. It is further assumed that subsystems are observable and have complex eigenvalues. Moreover, only one of the modes is allowed to be active on the switching plane. In this setup, the authors searched for periodic solutions which would be equivalent to searching for invariant cones in the original BPLS. The basic differences between the results presented in this paper and the works of Carmona et al. [20-22] can be summarized as follows. In this paper we use natural geometry induced by BPLS in $\mathbb{R}^{3}$. Furthermore, both subsystems are allowed to be active on the switching plane and vector field is not necessarily continuous. Therefore, the approach used by Carmona et al., [20,21] and the approach used in this paper are very different. The main result of Carmona et al., [20, Theorem 2] provides sufficient conditions for GAS of bimodal systems in terms of eigenvalues of subsystems, whereas in this paper a verifiable 
set of necessary and sufficient conditions for GAS of BPLS are provided. Carmona et al. [20-22] also investigate BPLS from a bifurcation point of view, whereas in this paper possible bifurcation sets are not considered. In a recent paper Carmona et al. [22] have also considered the case where both subsystems are unobservable and share the same real eigenvalue. They further assumed that the unobservable subspace has dimension one. It can be easily seen that these assumptions reduce the problem in $\mathbb{R}^{3}$ to $\mathbb{R}^{2}$. In fact, the necessary and sufficient conditions for GAS of BPLS in $\mathbb{R}^{3}$ given in [22] are actually equivalent to the necessary and sufficient conditions for the GAS of BPLS in $\mathbb{R}^{2}$ and are initially introduced by [24,23]. The same problem is considered in detail and in a more general setting in [13] where the vector field is allowed to be discontinuous, subsystems are observable and do not necessarily share the same real eigenvalue.

Iwatani and Hara [23] have also investigated BPLS. Theorem 19 of Iwatani and Hara [23], presents separate necessary and sufficient conditions for GAS of BPLS in $\mathbb{R}^{n}$ where $n>2$. The necessary condition given in Theorem 19 of [23] is obvious since it requires the negativity of the real eigenvalues. On the other hand, the sufficient condition in the same theorem is very restrictive, because it requires that the observability index of one of the subsystems is less than or equal to two. This result cannot be used in our framework, because in our setup the observability indexes of both subsystems are equal to three.

In a recent paper, Eren Y. et al. [15], introduced necessary and sufficient conditions for quadratic stability of BPLS in $\mathbb{R}^{n}$ where it is assumed that the vector field is continuous on the switching plane. The conditions are given in terms of LMI's (linear matrix inequalities) which consist of convex combinations of subsystem matrices (see Theorem 3 item 2 in [15]). In this context, the authors also provide the necessary and sufficient conditions for quadratic feedback stabilization (see Theorem 6 in [15]). Since quadratic stability is equivalent to the existence of a common quadratic Lyapunov function for subsystems, it is radically different from GAS.

Eldem and Sahan [13] also provides a verifiable set of necessary and sufficient conditions for GAS of BPLS in $\mathbb{R}^{3}$ with a simple geometry. They also assume that BPLS is well posed in which case the vector field is allowed to be discontinuous on the switching plane. It is assumed in [13] that the planes spanned by complex eigenvectors of each mode intersect in a line on the switching plane. Then, GAS of such BPLS was investigated for all possible combination of eigenvalues of both subsystems. It was shown that for each case, the solvability conditions of GAS of BPLS in $\mathbb{R}^{3}$ reduces down to the solvability conditions of GAS of BPLS in $\mathbb{R}^{2}$. This is expected intuitively, because the geometric configuration in this case is like folding $\mathbb{R}^{2}$ about the line separating the two modes of BPLS in $\mathbb{R}^{2}$ and placing it on $\operatorname{ker} \mathbf{c}^{T}$ in $\mathbb{R}^{3}$. In this paper, we assume that the planes spanned by complex eigenvectors of each mode intersect the switching plane in separate lines and the geometry induced in this case is much more complicated.

Eldem and Öner [14] provided a verifiable set of necessary and sufficient conditions for GAS of certain classes of BPLS. The work which is the closest one to the main result of this paper is [14]. One of the subsystems of BPLS considered in [14] has only real eigenvalues with algebraic degree equal to three and geometric degree equal to one. In this setup, it is shown that there exists a unique pair of fixed directions (to be defined later in the paper) which is attractive in a finite interval. In this paper, we show that there are two pairs of fixed directions, where one pair is attractive and the other pair is repulsive. In this respect, the class of BPLS considered in this paper exhibit a richer dynamic behavior than the class of BPLS considered in [14]. As in this paper, Eldem and Öner [14] also demonstrate the effect of the discontinuity of the vector field on the switching plane on GAS of BPLS being considered. Thus, the main result of this paper may be viewed as the extension of the results of [14] to a larger class of BPLS in $\mathbb{R}^{3}$.

The paper is organized as follows. In Section 2, BPLS to be considered in the paper is presented first. Then, the definitions and assumptions related to the issue of well-posedness of BPLS are given. It is further assumed that both modes have complex eigenvalues. The last part of Section 2 (Sections 2.1-2.5) is used to summarize some of the results from the literature. These subsections describe the behavior and the classification of the trajectories of BPLS before and after mode changes. Since these results constitute the foundations of our approach, they are necessary to present the main results of the paper. Main results are given in Section 3. An example which demonstrates the effect of the discontinuity of the vector field on GAS of BPLS being considered is also given in Section 3. Section 4 is on conclusions and future work.

\section{Preliminary results}

In the rest of the paper "solutions" and "trajectories" are used synonymously. The solutions means forward Caratheodory solutions (as defined in [8]) of the following BPLS

$\Sigma_{0}: \dot{\mathbf{x}}(t)=\left\{\begin{array}{ll}\mathbf{A}_{1} \mathbf{x}(t) & \text { if } \mathbf{c}^{T} \mathbf{x}(t) \geq 0 \\ \mathbf{A}_{2} \mathbf{x}(t) & \text { if } \mathbf{c}^{T} \mathbf{x}(t) \leq 0\end{array}\right\}$

where $\mathbf{x}, \mathbf{c} \in \mathbb{R}^{3}, \mathbf{A}_{1}$ and $\mathbf{A}_{2}$ are matrices in $\mathbb{R}^{3 \times 3}$. In this work, we consider the case where there is one real eigenvalue and a conjugate pair of imaginary eigenvalues in both modes. Thus, our next assumption is as follows.

Assumption 1 (A1): The eigenvalues of $A_{i}$ 's are $\left\{\lambda_{i}, \sigma_{i} \pm j w_{i}\right\}$, where $\lambda_{i}, \sigma_{i}$ and $w_{i}$ are real numbers and $w_{i}>0$.

\subsection{Well posedness}

Well posedness (existence and uniqueness of the solutions of $\Sigma_{0}$ ) is the first issue to resolve in such systems. Along this line, observe that the switching plane

$\mathscr{H}:=\left\{\mathbf{x}(t): \mathbf{c}^{T} \mathbf{x}(t)=\mathbf{0}\right\}$

divides $\mathbb{R}^{3}$ into two open half-spaces as described below.

$\mathscr{H}^{+}:=\left\{\mathbf{x}(t): \mathbf{c}^{T} \mathbf{x}(t)>\mathbf{0}\right\}$ and $\mathscr{H}^{-}:=\left\{\mathbf{x}(t): \mathbf{c}^{T} \mathbf{x}(t)<\mathbf{0}\right\}$. (3)

Thus, in view of the theory of differential equations, for any initial condition $\mathbf{x}_{0}$ in $\mathscr{H}^{+}$(or in $\mathscr{H}^{-}$) there exists a unique solution of $\Sigma_{0}$. However, since both modes are allowed to be active on the plane $\mathscr{H}$, the existence and uniqueness is not guaranteed. This issue is first resolved in [9]. Later, an alternative set of conditions on well posedness of BPLS in $\mathbb{R}^{3}$ is given in [13]. Finally, in [10], it was shown that well posedness introduce a joint structure for subsystem matrices of BPLS and BPAS (bimodal piecewise affine systems), if it is assumed that

Assumption2 (A2): The pairs $\left(\mathbf{c}^{T}, \mathbf{A}_{1}\right)$ and $\left(\mathbf{c}^{T}, \mathbf{A}_{2}\right)$ are observable and only $\left(\mathbf{c}^{T}, \mathbf{A}_{2}\right)$ is in observable canonical form.

In view of the above, we assume that

Assumption3 (A3): BPLS given by Eq. (1) is well posed.

With this last assumption and in view of Lemma 1 in [13] or in view of the first two items of Theorem 3.1 in [10], the components of $\Sigma_{0}$ can be written as follows.

$$
\begin{aligned}
& \mathbf{A}_{1}=\left[\begin{array}{ccc}
a_{11} & a_{12} & a_{13} \\
a_{21} & a_{22} & a_{23} \\
0 & a_{32} & a_{33}
\end{array}\right], \\
& \mathbf{A}_{2}=\left[\begin{array}{llc}
0 & 0 & \lambda_{2}\left(\sigma_{2}^{2}+w_{2}^{2}\right) \\
1 & 0 & -2 \sigma_{2} \lambda_{2}-\left(\sigma_{2}^{2}+w_{2}^{2}\right) \\
0 & 1 & 2 \sigma_{2}+\lambda_{2}
\end{array}\right], \\
& \mathbf{c}^{T}=\left[\begin{array}{lll}
0 & 0 & 1
\end{array}\right]
\end{aligned}
$$




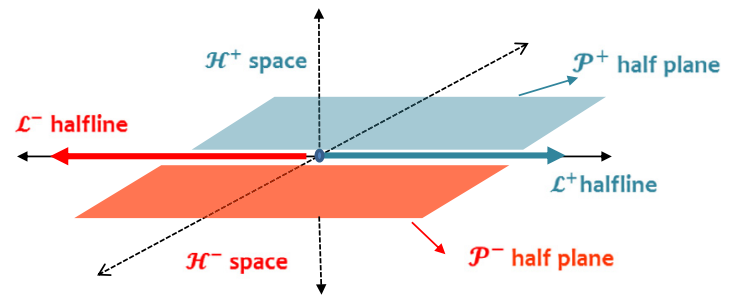

Fig. 1. Well posedness of BPLS.

where $a_{32}$ and $a_{21}$ are strictly positive. In this framework the vector field is allowed to be discontinuous on $\mathcal{H}$ and this fact distinguishes our work from some of the works in the literature, [20-22,24,15].

As shown in [13, Lemma 1], the joint structure of subsystem matrices directly implies and implied by the following.

- $\operatorname{ker} \mathbf{c}^{T} \cap \operatorname{ker}\left(\mathbf{c}^{T} \mathbf{A}_{1}\right)=\operatorname{ker} \mathbf{c}^{T} \cap \operatorname{ker}\left(\mathbf{c}^{T} \mathbf{A}_{2}\right)$ (or equivalently $a_{31}=0$ ).

- $a_{32}$ and $a_{21}>0$.

Note that the conditions given above, guarantee that the vector field of both modes have the same sign for any initial condition $\mathbf{x}_{0} \in \mathcal{H}$. In fact, if we define the line $\mathcal{L}$ as follows $\mathcal{L}:=\operatorname{ker} \mathbf{c}^{T} \cap$ $\operatorname{ker}\left(\mathbf{c}^{T} \mathbf{A}_{1}\right)=\operatorname{ker} \mathbf{c}^{T} \cap \operatorname{ker}\left(\mathbf{c}^{T} \mathbf{A}_{2}\right)$, then $\mathcal{L}$ divides $\mathscr{H}$ into two open half-planes defined as $\mathbf{c}^{T} \mathbf{A}_{i} \mathbf{x}_{0}>0,\left(\mathcal{P}^{+}\right)$and $\mathbf{c}^{T} \mathbf{A}_{i} \mathbf{x}_{0}<0,\left(\mathcal{P}^{-}\right)$ for $i=1,2$. Similarly, the origin divides $\mathcal{L}$ into two open half lines $\mathbf{c}^{T} \mathbf{A}_{i}^{2} \mathbf{x}_{0}>0,\left(\mathcal{L}^{+}\right)$and $\mathbf{c}^{T} \mathbf{A}_{i}^{2} \mathbf{x}_{0}<0,\left(\mathcal{L}^{-}\right)$on the right hand and left hand side of the origin, respectively.

In order to be consistent with the notation used in [9], let $\delta_{i}$ denote the set of initial conditions in $\mathbb{R}^{3}$ where a solution starts and continues in mode $i$, for $i=1,2$. Then, it follows that

$\varsigma_{1}=\mathscr{H}^{+} \cup \mathcal{P}^{+} \cup \mathcal{L}^{+}$and $\varsigma_{2}=\mathscr{H}^{-} \cup \mathcal{P}^{-} \cup \mathcal{L}^{-}$.

Furthermore, we have $\varsigma_{1} \cup \wp_{2}=\mathbb{R}^{3}$ and $\wp_{1} \cap \wp_{2}=\{\mathbf{0}\}$, which are equivalent to well posedness conditions given in [9]. This geometry is depicted in Fig. 1.

\subsection{The choice of eigenvectors and the behavior of the trajectories}

Let $\left\{\mathbf{r}_{i}\right\}$ and $\left\{\mathbf{x}_{i} \pm j \mathbf{y}_{i}\right\}(i=1,2)$ denote the real and complex eigenvectors of $\mathbf{A}_{i}$, respectively. If a trajectory starts in $s_{i}$ and continues in $\delta_{i}$ at least for some finite time, then it is convenient to express the initial conditions in $\delta_{i}$ as a linear combination of the eigenvectors of mode $i$. Then, the obvious choice for eigenvectors is as shown in [13, Lemma 2] and is given below.

$\mathbf{c}^{T} \mathbf{x}_{i}=\mathbf{c}^{T} \mathbf{r}_{i}=(-1)^{i+1}, \quad \mathbf{c}^{T} \mathbf{y}_{i}=0$,

$\mathbf{c}^{T} \mathbf{A}_{1} \mathbf{y}_{1}>0$ and $\mathbf{c}^{T} \mathbf{A}_{2} \mathbf{y}_{2}<0, \quad(i=1,2)$.

In this case it is straightforward to show that

$\mathbf{r}_{1}=\left[\begin{array}{c}\frac{\left(a_{11}-\sigma_{1}\right)^{2}+w_{1}^{2}+a_{12} a_{21}}{a_{32} a_{21}} \\ \frac{\lambda_{1}-a_{33}}{a_{32}} \\ 1\end{array}\right]$,
$\mathbf{x}_{1}=\left[\begin{array}{c}\frac{\left(a_{11}-\lambda_{1}\right)\left(a_{11}-\sigma_{1}\right)+a_{12} a_{21}}{a_{32} a_{21}} \\ \frac{\sigma_{1}-a_{33}}{a_{32}} \\ 1\end{array}\right]$,

$\mathbf{y}_{1}=\left[\begin{array}{c}\frac{w_{1}\left(a_{11}-\lambda_{1}\right)}{a_{32} a_{21}} \\ \frac{w_{1}}{a_{32}} \\ 0\end{array}\right]$, and

$\mathbf{r}_{2}:=\left[\begin{array}{c}-\left(\sigma_{2}^{2}+w_{2}^{2}\right) \\ 2 \sigma_{2} \\ -1\end{array}\right]$ and

$\left[\begin{array}{ll}\mathbf{x}_{2} & \mathbf{y}_{2}\end{array}\right]=\left[\begin{array}{cc}-\sigma_{2} \lambda_{2} & w_{2} \lambda_{2} \\ \left(\sigma_{2}+\lambda_{2}\right) & -w_{2} \\ -1 & 0\end{array}\right]^{T}$.

Thus, we can use two different bases for $\mathbb{R}^{3}$. The set of trajectories that start out with initial conditions $\delta_{i} \mathbf{r}_{i}$ where $\delta_{i}>0$ will decay to the origin without going into the other mode if and only if $\lambda_{i}<0$. Therefore, we need to investigate only the trajectories with nontrivial sinusoidal parts. More specifically, let $\mathbf{z}_{i}(t)(i=1,2)$ denote the trajectories which start from $\delta_{i}$ and continue in $\delta_{i}$. Then, the behavior of such trajectories in the $i$ th mode can be written as follows.

$$
\begin{aligned}
\mathbf{z}_{i}(t)= & K_{i}\left\{\alpha_{i} \exp \left(\lambda_{i} t\right) \mathbf{r}_{i}+\exp \left(\sigma_{i} t\right)\left(\sin \left(\theta_{i}+w_{i} t\right) \mathbf{x}_{i}\right.\right. \\
& \left.\left.+\cos \left(\theta_{i}+w_{i} t\right) \mathbf{y}_{i}\right)\right\}, \quad \text { for } i=1,2 .
\end{aligned}
$$

This implies that

$$
\begin{aligned}
\mathbf{c}^{T} \mathbf{z}_{i}(t) & =K_{i}\left\{\alpha_{i} \exp \left(\lambda_{i} t\right)+\exp \left(\sigma_{i} t\right) \sin \left(\theta_{i}+w_{i} t\right)\right\} \\
& =K_{i} \exp \left(\lambda_{i} t\right)\left\{f_{i}(t)\right\}
\end{aligned}
$$

where

$f_{i}(t):=\alpha_{i}+\exp \left(\left(\sigma_{i}-\lambda_{i}\right) t\right) \sin \left(\theta_{i}+w_{i} t\right)$.

See [13, Lemma 2] for details. Note that the sign of $f_{i}(t)$ and the sign of $\mathbf{c}^{T} \mathbf{z}_{i}(t)$ are the same. Furthermore, it is clear that $\theta_{i}$ is the angle on the plane spanned by $\left\{\mathbf{x}_{i}, \mathbf{y}_{i}, i=1,2\right\}$ and it is employed to indicate the linear combinations of $\left\{\mathbf{x}_{i}, \mathbf{y}_{i}, i=1,2\right\}$. Hence, the domain of $\theta_{i}$ is $[0,2 \pi]$.

Note that trajectories which start and continue in $\delta_{i}$ may or may not change mode in finite time. Since this is a crucial issue for GAS, a natural step forward is the following classification.

\subsection{Classification of transitive and nontransitive trajectories}

Transitive and nontransitive trajectories are defined as follows.

Definition 1 ([13, Definition 1$])$. A trajectory $\mathbf{z}_{i}\left(\tau_{i}\right)$ of $\Sigma_{0}$ given by Eq. (8) is called transitive if there exists a finite $\tau_{i}>0$ such that $c^{T} z_{i}\left(\tau_{i}\right)=0$ and the trajectory changes mode at $t=\tau_{i}$. Otherwise, it is called nontransitive.

Remark 1. Eqs. (9)-(11) can be used for the classification given above. In order to simplify the notation let us define

$\hat{\mathbf{x}}_{i}:=\mathbf{x}_{i}-\mathbf{r}_{i}$ and $\quad b_{i}:=\cot \phi_{i}:=\frac{\sigma_{i}-\lambda_{i}}{w_{i}}$ for $i=1,2$.

If a trajectory $\mathbf{z}_{i}(t)$ starts from $\mathcal{H}$, then $\alpha_{i}=-\sin \left(\theta_{i}\right)$ in Eq. (11). Consequently, we get $\mathbf{z}_{i}(0)=K_{i}\left(\sin \left(\theta_{i}\right) \hat{\mathbf{x}}_{i}+\cos \left(\theta_{i}\right) \mathbf{y}_{i}\right)$ where $K_{i}>0$. Thus, $\hat{\mathbf{x}}_{i}$ and $\mathbf{y}_{i}$ constitute a basis for $\mathscr{H}$ for $i=$ 1 , 2. Furthermore, it is straightforward to show that $\mathbf{c}^{T} \mathbf{A}_{i} \mathbf{z}_{i}(t)=$ $K_{i}(-1)^{i+1} M_{i} \sin \left(\theta_{i}+w_{i} t+\phi_{i}\right)$ for $t \geq 0$ where

$M_{1}:=\frac{\left(\left(\sigma_{1}-\lambda_{1}\right)^{2}+w_{1}^{2}\right)^{1 / 2}}{a_{32}}$, and

$M_{2}:=\left(\left(\sigma_{2}-\lambda_{2}\right)^{2}+w_{2}^{2}\right)^{1 / 2}$.

It can also be shown easily (see the proof of Lemma 3 in [13]) that if $\theta_{i}=-\phi_{i}$, then $\mathbf{c}^{T} \mathbf{z}_{i}(0)=0$ and $\mathbf{c}^{T} \mathbf{A}_{i} \mathbf{z}_{i}(0)=0$, but $\mathbf{c}^{T} \mathbf{A}_{i}^{2} \mathbf{z}_{i}(0)>0$ for $i=1$ and $\mathbf{c}^{T} \mathbf{A}_{i}^{2} \mathbf{z}_{i}(0)<0$ for $i=2$. In view of these observations any initial condition $\mathbf{z}_{i}(0)=K_{i}\left(\sin \left(\theta_{i}\right) \hat{\mathbf{x}}_{i}+\cos \left(\theta_{1}\right) \mathbf{y}_{i}\right)$ smoothly 
continues in $\gamma_{i}$ if and only if $-\phi_{i} \leq \theta_{i}<\pi-\phi_{i}$, because the vector fields of both modes point into $\wp_{i}$. Note that if $\pi-\phi_{i} \leq \theta_{i}<2 \pi-\phi_{i}$, then smooth continuation into $\gamma_{i}$ is not possible, because the vector fields of both modes point into the other mode. After these preliminary remarks, the trajectories of each mode can be classified as described in [13].

Lemma 1 ([13, Lemma 4]). Consider the system $\Sigma_{0}$ described by Eqs. (1) and (4). Suppose that $\mathbf{z}_{i}(0) \in \mathscr{H}$. Then, the following hold.

(1) If $\sigma_{i}-\lambda_{i}>0$, then all possible trajectories $z_{i}(t)$ are transitive.

(2) If $\sigma_{i}-\lambda_{i} \leq 0$, then there exists a unique angle $\varphi_{i} \in\left(0, \phi_{i}\right]$ such that

(a) If $\theta_{i} \in\left[-\phi_{i},-\varphi_{i}\right]$ and $\mathbf{z}_{i}(0)=K_{i}\left(\sin \left(\theta_{i}\right) \hat{\mathbf{x}}_{i}+\cos \left(\theta_{i}\right) \mathbf{y}_{i}\right)$, then these trajectories are nontransitive,

(b) If $\theta_{i} \in\left(-\varphi_{i}, \pi-\phi_{i}\right)$ and $\mathbf{z}_{i}(0)=K_{i}\left(\sin \left(\theta_{i}\right) \hat{\mathbf{x}}_{i}+\cos \left(\theta_{i}\right) \mathbf{y}_{i}\right)$, then these trajectories are "transitive".

In view of the above result, let $\mathbf{V}_{i}\left(\theta_{i}\right):=\sin \left(\theta_{i}\right) \hat{\mathbf{x}}_{i}+\cos \left(\theta_{i}\right) \mathbf{y}_{i}$. Following the terminology used in [13], we refer to $\mathbf{V}_{i}\left(\theta_{i}\right)$ as directions. It is clear that if $\mathbf{V}_{1}\left(\theta_{1}\right)$ is a direction, then there exists $\theta_{2}$ and a constant $\eta_{12}\left(\theta_{1}\right)>0$ such that

$\mathbf{V}_{1}\left(\theta_{1}\right)=\eta_{12}\left(\theta_{1}\right) \mathbf{V}_{2}\left(\theta_{2}\right)$.

In this case we shall refer to $\mathbf{V}_{1}\left(\theta_{1}\right)$ and $\mathbf{V}_{2}\left(\theta_{2}\right)$ as "equivalent directions" and use the notation $\mathbf{V}_{1}\left(\theta_{1}\right) \simeq \mathbf{V}_{2}\left(\theta_{2}\right)$. Furthermore, we shall also use the terminology "a trajectory starting from direction $\mathbf{V}_{i}\left(\theta_{i}\right)$ " in order to refer to a trajectory starting on $\mathscr{H}$ with initial condition $\mathbf{z}_{i}(0)=K_{i} \mathbf{V}_{i}\left(\theta_{i}\right)$ where $K_{i}>0$ is a real constant.

Remark 2. Note that when $\sigma_{i}-\lambda_{i} \leq 0$, the corresponding mode will be stable if $\lambda_{i}<0$. Then, any trajectory which starts with initial condition $K_{i} \mathbf{V}_{i}\left(\theta_{i}\right)$ where $\theta_{i} \in\left[-\phi_{i},-\varphi_{i}\right]$ and $K_{i}>0$ will stay in the same mode and decay to the origin. Also note that the set of such initial conditions constitute a cone in $\mathscr{H}$ bounded by the directions $\mathbf{V}_{i}\left(-\phi_{i}\right)$ and $\mathbf{V}_{i}\left(-\varphi_{i}\right)$. We formalize this observation by the following definition.

Definition 2 ([13, Definition 2]). If $\sigma_{i}-\lambda_{i} \leq 0$ for a mode $i$, then the closed convex cone in $\mathscr{H} \cap \delta_{i}$ bounded by the directions $\mathbf{V}_{i}\left(-\phi_{i}\right)$ and $\mathbf{V}_{i}\left(-\varphi_{i}\right)$ will be called mode-invariant cone $\mathcal{C}_{i}$ of $\mathscr{H} \cap s_{i}$.

Remark 3. Recall that (Definition 1 ) if $\mathbf{z}_{i}(t)$ is a transitive trajectory, then there exists $\tau_{i}>0$ such that $f_{i}\left(\tau_{i}\right)=0$, where $f_{i}(t)$ is as given in Eq. (11). This implies that $\tau_{i}$ is a function of $\theta_{i}$ and Eq. (11) implies that this functional relationship can be written implicitly as follows.

$-\sin \theta_{i}+\exp \left(\left(\sigma_{i}-\lambda_{i}\right) \tau_{i}\right) \sin \left(\theta_{i}+w_{i} \tau_{i}\right)=0$.

In view of this equation, $\sin \theta_{i}$ and $\sin \left(\theta_{i}+w_{i} \tau_{i}\right)$ must have the same sign for transitive trajectories. Furthermore, as explained in Remark 1, $\mathbf{c}^{T} \mathbf{A}_{1} \mathbf{z}(t)>0$ if $-\phi_{1} \leq \theta_{1}+w_{1} t<\pi-\phi_{1}$. Consequently, if $\theta_{1}>0$, then the curves $\sin \theta_{1} \exp \left(-\left(\sigma_{1}-\lambda_{1}\right) t\right)$ and $\sin \left(\theta_{1}+w_{1} t\right)$ intersect when $\pi-\phi_{1}<\theta_{1}+w_{1} t<\pi$. If $\sigma_{i}-\lambda_{i}>0$ and $-\phi_{1} \leq \theta_{1} \leq 0$, then the intersection occurs when $\pi \leq \theta_{1}+w_{1} t<\pi+\phi_{1}$. This is illustrated in Fig. 2 .

On the other hand, if $\sigma_{i}-\lambda_{i} \leq 0$ and $-\varphi_{1}<\theta_{1} \leq 0$, then the intersection occurs when $\pi \leq \theta_{1}+w_{1} t<2 \pi-\phi_{1}$. This intersection point is $t=\tau_{1}$ where the trajectory hits $\mathscr{H}$ and changes mode. (See Fig. 3.)

In view of these observations, it is clear that $\tau_{i}$ and the map $\mathcal{F}_{i}: \theta_{i} \rightarrow \theta_{i}+w_{i} \tau_{i}$ are important in describing the behavior of trajectories as they evolve in $\oiint_{i}$. Properties of $\tau_{i}$ and $\mathcal{F}_{i}$ are given by the following result in [13].

Lemma 2 ([13, Lemma 5-6]). Consider BPLS given by Eqs. (1) and (4) and let $z_{i}(t)$ be a transitive trajectory with $z_{i}(0) \in s_{i} \cap \mathcal{H}$. Then, the following hold for the time $\tau_{i}$ at which the trajectory changes mode.

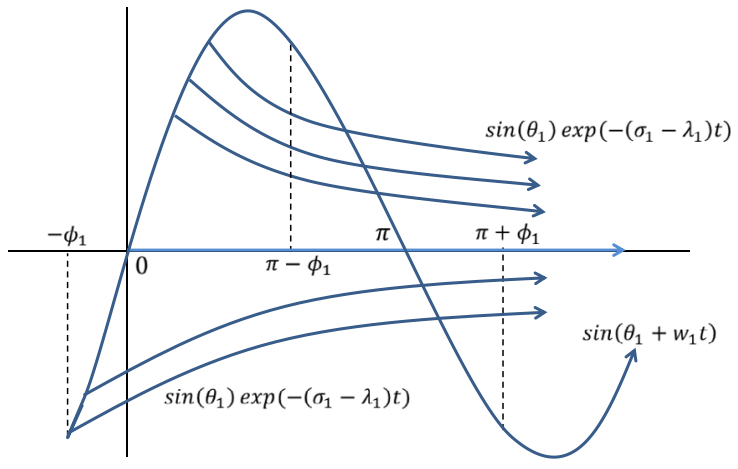

Fig. 2. Intersection points for $\sigma_{i}-\lambda_{i}>0$.

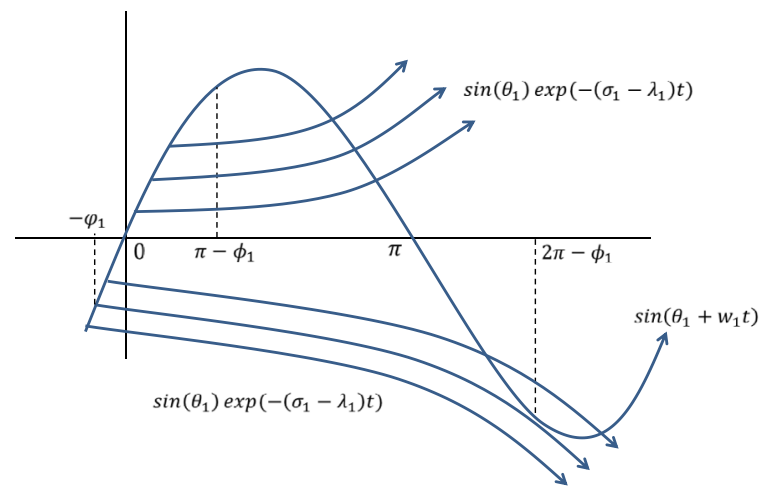

Fig. 3. Intersection points for $\sigma_{i}-\lambda_{i} \leq 0$.

(1) $\tau_{i}\left(\theta_{i}\right)$ and $\theta_{i}+w_{i} \tau_{i}\left(\theta_{i}\right)$ are decreasing functions of $\theta_{i}$ and

$$
\begin{aligned}
& \lim _{\theta_{i} \rightarrow \pi-\phi_{i}} \tau_{i}\left(\theta_{i}\right)=0 \text { and } \\
& \lim _{\theta_{i} \rightarrow 0 \pm} \frac{d\left(\theta_{i}+w_{i} \tau_{i}\left(\theta_{i}\right)\right)}{d \theta_{i}}=-\exp \left(-b_{i} \pi\right) .
\end{aligned}
$$

(2) Let $\mathcal{F}_{i}: \theta_{i} \rightarrow \theta_{i}+w_{i} \tau_{i}\left(\theta_{i}\right)$. Then $\frac{d \mathcal{F}_{i}\left(\theta_{i}\right)}{d \theta_{i}}=\frac{\cot \left(\theta_{i}\right)+b_{i}}{\cot \left(\theta_{i}+w_{i} \tau_{i}\left(\theta_{i}\right)\right)+b_{i}} \leq 0$ and the following hold.

(a) If $\sigma_{i}-\lambda_{i}>0$, then $\mathcal{F}_{i}$ is a nonexpansive map i.e., $\sup _{-\phi_{i} \leq \theta_{i}<\pi-\phi_{i}}\left|\frac{d F_{i}}{d \theta_{i}}\right| \leq 1$ and $\lim _{\theta_{i} \rightarrow \pi-\phi_{i}} \frac{d F_{i}}{d \theta_{i}}=-1$,

(b) If $\sigma_{i}-\lambda_{i} \leq 0$, then $\mathcal{F}_{i}$ is an expansive map.

$$
\begin{aligned}
& \inf _{-\varphi_{i}<\theta_{i}<\pi-\phi_{i}}\left|\frac{d F_{i}}{d \theta_{i}}\right| \geq 1, \quad \lim _{\theta_{i} \rightarrow \pi-\phi_{i}} \frac{d F_{i}}{d \theta_{i}}=-1, \quad \text { and } \\
& \lim _{\theta_{i} \rightarrow\left(-\varphi_{i}\right)^{+}} \frac{d F_{i}}{d \theta_{i}}=-\infty .
\end{aligned}
$$

(c) If $\sigma_{i}-\lambda_{i}=0$, then $\varphi_{i}=\frac{\pi}{2}$ and $\frac{d F_{i}}{d \theta_{i}}=-1$.

Remark 4. Note that $\mathcal{F}_{i}\left(\theta_{i}\right)$ is not defined at $\theta_{i}=\pi-\phi_{i}$, because smooth continuation in mode $i$ is not possible at $\theta_{i}=\pi-\phi_{i}$ (smooth continuation is possible in the other mode at $\theta_{i}=\pi-\phi_{i}$ ). However, in view of Lemma 2(1), we can define $\tau_{i}\left(\pi-\phi_{i}\right):=0$ and $\mathcal{F}_{i}\left(\pi-\phi_{i}\right):=\pi-\phi_{i}$ and "continuously extend" $\mathcal{F}_{i}\left(\theta_{i}\right)$ to $\theta_{i}=\pi-\phi_{i}$. Thus, it follows that $\mathcal{F}_{i}\left(\theta_{i}\right)$ is a continuous function defined over the closed interval $\left[0, \pi-\phi_{i}\right]$.

In order to simplify the notation, we use $\tau_{i}$ instead of $\tau_{i}\left(\theta_{i}\right)$ (to denote the time at which the trajectories change mode), in the rest of the paper.

\subsection{Mode change and a further classification of trajectories}

In the previous subsection we have defined directions $V_{i}\left(\theta_{i}\right)$ as vectors in $\mathcal{H}$, expressed as $V_{i}\left(\theta_{i}\right):=\sin \left(\theta_{i}\right) \hat{\mathbf{x}}_{i}+\cos \left(\theta_{i}\right) \mathbf{y}_{i}$. Let 
$\left[\begin{array}{ll}\Gamma_{i}^{T} & 0\end{array}\right]^{T}:=\left[\begin{array}{ll}\hat{\mathbf{x}}_{i} & \mathbf{y}_{i}\end{array}\right]$. Then using Eqs. (7), (8), and (12), we get

$\boldsymbol{\Gamma}_{1}=\left[\begin{array}{cc}\frac{\left(a_{11}-\sigma_{1}\right)\left(\sigma_{1}-\lambda_{1}\right)-w_{1}^{2}}{a_{32} a_{21}} & \frac{w_{1}\left(a_{11}-\lambda_{1}\right)}{a_{32} a_{21}} \\ \frac{\sigma_{1}-\lambda_{1}}{a_{32}} & \frac{w_{1}}{a_{32}}\end{array}\right]$ and

$\boldsymbol{\Gamma}_{2}=\left[\begin{array}{cc}\sigma_{2}\left(\sigma_{2}-\lambda_{2}\right)+\omega_{2}^{2} & w_{2} \lambda_{2} \\ -\left(\sigma_{2}-\lambda_{2}\right) & -w_{2}\end{array}\right]$

where $\boldsymbol{\Gamma}_{1}$ and $\boldsymbol{\Gamma}_{2}$ are clearly nonsingular. When a trajectory changes mode, we have to change to the relevant basis in order to follow the behavior of the trajectories. This is possible by using the relation between equivalent directions given in Eq. (13). This relation can be written explicitly as follows.

$\left[\begin{array}{c}\sin \theta_{1} \\ \cos \theta_{1}\end{array}\right]=\eta_{12}\left(\theta_{1}\right) \boldsymbol{\Gamma}_{1}^{-1} \boldsymbol{\Gamma}_{2}\left[\begin{array}{c}\sin \theta_{2} \\ \cos \theta_{2}\end{array}\right]$.

The solution of this equation yields $\eta_{12}\left(\theta_{1}\right)$ and $\theta_{2}$ as functions of $\theta_{1}$. Towards this end, we first define

$\mathscr{B}_{1}:=\frac{a_{11}-\lambda_{1}+a_{21} \lambda_{2}}{a_{21} w_{2}\left(1+b_{2}^{2}\right)} \quad$ and $\quad C_{1}:=\frac{w_{1}\left(1+b_{1}^{2}\right)}{a_{21} w_{2}\left(1+b_{2}^{2}\right)}$,

where $\mathscr{B}_{1}$ is called "the coupling constant". The solution of Eq. (13) is given by the results presented below.

Lemma 3 ([13, Lemma 7]). Let $V_{1}\left(\theta_{1}\right)$ be a direction. Then, there exists a unique $\theta_{2} \in\left[-\phi_{2}, 2 \pi-\phi_{2}\right)$ and $\eta_{12}\left(\theta_{1}\right)>0$ such that $V_{1}\left(\theta_{1}\right)=\eta_{12}\left(\theta_{1}\right) V_{2}\left(\theta_{2}\right)$ where

$\theta_{2}=\cot ^{-1}\left\{\frac{\left(\cot \theta_{1}+b_{1}\right)}{C_{1}-B_{1}\left(\cot \theta_{1}+b_{1}\right)}-b_{2}\right\}$,

$\eta_{12}\left(\theta_{1}\right)=\frac{-w_{1} \sin \left(\theta_{1}+\phi_{1}\right)}{a_{32} w_{2} \sin \left(\theta_{2}+\phi_{2}\right)} \frac{\sin \phi_{2}}{\sin \phi_{1}}$,

for $\theta_{1} \in\left(-\phi_{1}, \pi-\phi_{1}\right) \cup\left(\pi-\phi_{1}, 2 \pi-\phi_{1}\right)$, and

$\eta_{12}\left(\theta_{1}\right)=C_{1} \frac{w_{1}}{a_{32} w_{2}} \frac{\sin \phi_{1}}{\sin \phi_{2}}$ for $\theta_{1}=-\phi_{1}, \pi-\phi_{1}$.

Along the line of the result given above, if a trajectory in $\delta_{2}$ changes mode, then there exists a unique $\theta_{1} \in\left[-\phi_{1}, 2 \pi-\phi_{1}\right)$ and $\eta_{21}\left(\theta_{2}\right)>0$ such that $V_{2}\left(\theta_{2}\right)=\eta_{21}\left(\theta_{2}\right) V_{1}\left(\theta_{1}\right)$. Then, in order to obtain $\theta_{1}$ and $\eta_{21}\left(\theta_{2}\right)$, one can follow the same lines as in the proof [13, Lemma 7] and easily show that

$\theta_{1}=\cot ^{-1}\left\{\frac{\left(\cot \theta_{2}+b_{2}\right)}{C_{2}+\mathscr{B}_{2}\left(\cot \theta_{2}+b_{2}\right)}-b_{1}\right\}$,

and

$\eta_{21}\left(\theta_{2}\right)=\frac{-a_{32} w_{2} \sin \left(\theta_{2}+\phi_{2}\right)}{w_{1} \sin \left(\theta_{1}+\phi_{1}\right)} \frac{\sin \phi_{1}}{\sin \phi_{2}}$

where $C_{2}:=\left(C_{1}\right)^{-1}, \mathcal{B}_{2}:=C_{2} \mathcal{B}_{1}$, and $\theta_{2} \in\left(-\phi_{2}, \pi-\phi_{2}\right) \cup$ $\left(\pi-\phi_{2}, 2 \pi-\phi_{2}\right)$. For $\theta_{2}=-\phi_{2}, \pi-\phi_{2}$, we get

$\eta_{21}\left(\theta_{2}\right)=C_{2} \frac{a_{32} w_{2}}{w_{1}} \frac{\sin \phi_{2}}{\sin \phi_{1}}$.

In view of the above developments we can now define $g_{i}\left(\theta_{i}\right)$ as follows.

$g_{1}\left(\theta_{1}\right)=\cot ^{-1}\left\{\frac{\left(\cot \theta_{1}+b_{1}\right)}{C_{1}-B_{1}\left(\cot \theta_{1}+b_{1}\right)}-b_{2}\right\}$
$g_{2}\left(\theta_{2}\right)=\cot ^{-1}\left\{\frac{\left(\cot \theta_{2}+b_{2}\right)}{C_{2}+\mathcal{B}_{2}\left(\cot \theta_{2}+b_{2}\right)}-b_{1}\right\}$.

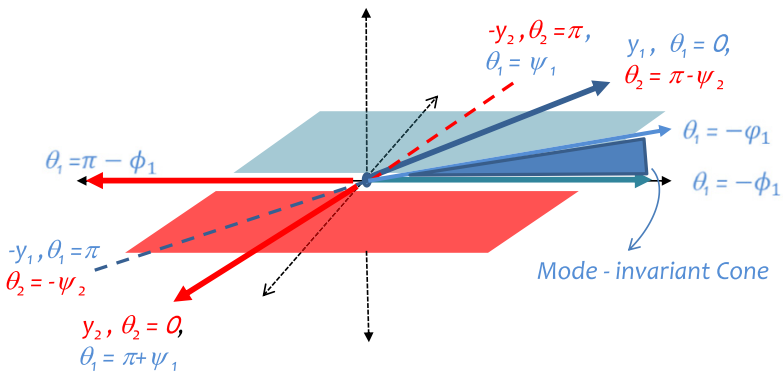

Fig. 4. Geometry of directions on $\mathscr{H}$.

Let $g_{1}\left(\pi-\psi_{1}\right):=0$ and $g_{2}\left(\pi+\psi_{2}\right):=0$. With these definitions $g_{i}\left(\theta_{i}\right)$ is well-defined and continuous over the real line. Using the expressions of $g_{i}\left(\theta_{i}\right)$, the following equivalent directions can be obtained easily.

$V_{1}\left(-\phi_{1}\right) \simeq V_{2}\left(\pi-\phi_{2}\right)$, and $V_{1}\left(\pi-\phi_{1}\right) \simeq V_{2}\left(-\phi_{2}\right)$.

$V_{1}\left(\psi_{1}\right) \simeq V_{2}(\pi)=-\mathbf{y}_{2}$, and $V_{1}(\pi) \simeq V_{2}\left(-\psi_{2}\right)=-\mathbf{y}_{1}$

where $\cot \psi_{2}:=\mathcal{B}_{1}^{-1}+b_{2}$, cot $\psi_{1}:=\mathcal{B}_{2}^{-1}-b_{1}$ (see Fig. 4).

Note that the functions $\mathcal{F}_{i}$ 's determine the angle at which a trajectory hits $\mathscr{H}$ and changes mode. On the other hand, the functions $g_{i}$ 's define the transformations between modes. In order to formalize what we have explained in the previous section, let

$\mathcal{T}_{1}\left(\theta_{1}\right):=g_{2}\left(\mathcal{F}_{2}\left(g_{1}\left(\mathcal{F}_{1}\left(\theta_{1}\right)\right)\right)\right)$.

Since $\mathcal{F}_{i}$ 's and $g_{i}$ 's are continuous, it follows that $\mathcal{T}_{1}$ is also continuous. Thus, we have

$\theta_{1} \stackrel{\mathcal{F}_{1}}{\rightarrow} \theta_{1}+w_{1} \tau_{1} \stackrel{g_{1}}{\rightarrow} \theta_{2} \stackrel{\mathcal{F}_{2}}{\rightarrow} \theta_{2}+w_{2} \tau_{2} \stackrel{g_{2}}{\rightarrow} \theta_{11}:=\mathcal{T}_{1}\left(\theta_{1}\right)$

Since some trajectories may change mode several times and then enter a mode-invariant cone, we now give the following classification.

(1) the trajectories which change mode finite number of times as $t \rightarrow \infty$

(2) the trajectories which change mode infinite number of times as $t \rightarrow \infty$.

The trajectories which change mode only a finite number of times decay to the origin if and only if $\lambda_{i}<0$ for both modes. In view of this observation, we need to investigate only GAS of the class of trajectories which change mode infinite number of times as $t \rightarrow \infty$. Since such trajectories change mode at $\mathscr{H}$, we can restrict our investigation to trajectories which start from $\mathscr{H}$ without any loss of generality.

In view of Remark 1 and Lemma 1 , the domain of $\mathcal{T}_{1}$ is $\left[-\phi_{1}, \pi-\right.$ $\left.\phi_{1}\right]$ if $\sigma_{1}-\lambda_{1}>0$. If $\sigma_{1}-\lambda_{1} \leq 0$, then the domain of $\mathcal{T}_{1}$ is $\left(-\varphi_{1}, \pi-\phi_{1}\right] . \mathcal{T}_{1}$ is defined for trajectories which change mode at least two times. For trajectories which change mode $2 k$ times, let $\mathcal{T}_{1}^{k}\left(\theta_{1}\right)$ denote

$\mathcal{T}_{1}^{k}\left(\theta_{1}\right):=\mathcal{T}_{1}\left(\ldots . \mathcal{T}_{1}\left(\mathcal{T}_{1}\left(\theta_{1}\right)\right) \ldots ..\right) \quad(k$ times $)$ and

$\theta_{1 k}:=\mathcal{T}_{1}^{k}\left(\theta_{1}\right)$.

We can also define $\mathcal{T}_{2}$ as $\mathcal{T}_{2}\left(\theta_{2}\right):=g_{1}\left(\mathcal{F}_{1}\left(g_{2}\left(\mathcal{F}_{2}\left(\theta_{2}\right)\right)\right)\right.$ ), which is again a continuous function and use the notation $\mathcal{T}_{2}^{k}\left(\theta_{2}\right)$ in a completely similar way. In order to investigate the trajectories which change mode infinite number of times as $t \rightarrow \infty$, we need the following definition.

Definition 3 ([13, Definition 3]). A direction $V_{i}\left(\theta_{i}^{*}\right)$ in $\mathscr{H}$ is called a fixed direction if $\theta_{i}^{*}$ is a fixed point of $\mathcal{T}_{i}\left(\theta_{i}\right)$, or equivalently $\mathcal{T}_{i}\left(\theta_{i}^{*}\right)=\theta_{i}^{*}$. A fixed direction $V_{i}\left(\theta_{i}^{*}\right)$ is called attractive on the interval $I$ which includes $\theta_{i}^{*}$, if for any $\theta_{i} \in I$ and any $\varepsilon>0$ there exists a positive integer $k$ such that we have $\left|\mathcal{T}_{i}^{k}\left(\theta_{i}\right)-\theta_{i}^{*}\right|<\varepsilon$. If $I$ consists of only one point $\theta_{i}^{*}$, then the fixed direction is called repulsive. 
Remark 5. If $V_{1}\left(\theta_{1}^{*}\right)$ is a fixed direction in $\mathscr{H} \cap \S_{1}$, then there exists $\theta_{2}^{*}:=g_{1}\left(\mathcal{F}_{1}\left(\theta_{1}^{*}\right)\right)$ such that $V_{2}\left(\theta_{2}^{*}\right)$ is a fixed direction in $\mathscr{H} \cap \varsigma_{2}$. Hence, fixed directions exist in pairs. In view of this observation, we shall also use the notation $V\left(\theta_{1}^{*}, \theta_{2}^{*}\right)$ to denote a pair of fixed directions in the sequel. Furthermore, if all the trajectories starting from a fixed direction are stable (decays to the origin as $t \rightarrow$ $\infty)$, then the fixed direction is called stable, or equivalently, fixed direction pair will be called stable.

Since the attractivity of mode-invariant cone $\mathcal{C}_{i}$ is also important in stability analysis we also need the following definition (which is slightly modified version of Definition 2 in [13]).

Definition 4. $\mathcal{C}_{i}$ is said to be attractive in an interval $I_{i}$ containing $C_{i}$ if for any $\theta_{i} \in I_{i}$ there exists a finite nonnegative integer $k$ such that $-\phi_{i} \leq \mathcal{T}_{i}{ }^{k}\left(\theta_{i}\right) \leq-\varphi_{i}$ or $\mathcal{T}_{i}{ }^{k}\left(\theta_{i}\right) \rightarrow-\varphi_{i}$ as $k \rightarrow \infty$. In the latter case, the boundary $V_{i}\left(-\varphi_{i}\right)$ will also be called degenerate fixed direction. If $I_{i}=\left[-\phi_{i},-\varphi_{i}\right]$, then $\mathcal{C}_{i}$ is said to be repulsive.

\subsection{Stability of fixed directions}

Let $\theta_{1}^{*}$ be a fixed point of $\mathcal{T}_{1}($.$) , i.e., \mathcal{T}_{1}\left(\theta_{1}^{*}\right)=\theta_{1}^{*}$ and $\mathbf{z}_{1}^{*}(t)$ be a trajectory starting from $\mathscr{H} \cap \varsigma_{1}$ with initial condition $\mathbf{z}_{1}^{*}(0)=$ $K_{1} \mathbf{V}_{1}\left(\theta_{1}^{*}\right)$. This trajectory is first mapped to $\mathscr{H} \cap \varkappa_{2}$. Then, in view of Lemma 3 and Eq. (13), we get

$$
\begin{aligned}
K_{1} \mathbf{V}_{1}\left(\theta_{1}^{*}\right) \rightarrow & K_{1} \exp \left(\sigma_{1} \tau_{1}^{*}\right) \mathbf{V}_{1}\left(\theta_{1}^{*}+w_{1} \tau_{1}^{*}\right) \\
\rightarrow & K_{1} \exp \left(\sigma_{1} \tau_{1}^{*}\right) \eta_{12}\left(\theta_{1}^{*}+w_{1} \tau_{1}^{*}\right) \mathbf{V}_{2}\left(\theta_{2}^{*}\right) \\
\rightarrow & K_{1} \exp \left(\sigma_{1} \tau_{1}^{*}+\sigma_{2} \tau_{2}^{*}\right) \eta_{12}\left(\theta_{1}^{*}+w_{1} \tau_{1}^{*}\right) \\
& \times \mathbf{V}_{2}\left(\theta_{2}^{*}+w_{2} \tau_{2}^{*}\right) \\
\rightarrow & K_{1} \exp \left(\sigma_{1} \tau_{1}^{*}+\sigma_{2} \tau_{2}^{*}\right) \eta_{12}\left(\theta_{1}^{*}+w_{1} \tau_{1}^{*}\right) \\
& \times \eta_{21}\left(\theta_{2}^{*}+w_{2} \tau_{2}^{*}\right) \mathbf{V}_{1}\left(\theta_{1}^{*}\right) .
\end{aligned}
$$

This is one loop about $\mathscr{H}$ and can be interpreted as a full Poincaré map and written as follows.

$K_{1} \mathbf{V}_{1}\left(\theta_{1}^{*}\right) \rightarrow \gamma_{F}\left(\theta_{1}^{*}\right) K_{1} \mathbf{V}_{1}\left(\theta_{1}^{*}\right)$

where the convergence rate $\gamma_{F}\left(\theta_{1}^{*}\right)$ of fixed direction $\mathbf{V}_{1}\left(\theta_{1}^{*}\right)$ is defined as follows.

$\gamma_{F}\left(\theta_{1}^{*}\right):=\exp \left(\sigma_{1} \tau_{1}^{*}+\sigma_{2} \tau_{2}^{*}\right) \eta_{12}\left(\theta_{1}^{*}+w_{1} \tau_{1}^{*}\right) \eta_{21}\left(\theta_{2}^{*}+w_{2} \tau_{2}^{*}\right)$.

Recall that fixed directions exist in pairs $\left(\theta_{2}^{*}:=g_{1}\left(\mathcal{F}_{1}\left(\theta_{1}^{*}\right)\right)\right)$. Therefore, the notation $\gamma_{F}\left(\theta_{1}^{*}, \theta_{2}^{*}\right)$ will also be used for convergence rate.

If the trajectory undergoes $k$ loops around $\mathscr{H}$, then we have

$K_{1} \mathbf{V}_{1}\left(\theta_{1}^{*}\right) \rightarrow\left(\gamma_{F}\left(\theta_{1}^{*}\right)\right)^{k} K_{1} \mathbf{V}_{1}\left(\theta_{1}^{*}\right)$.

In view of the above equation, it follows that a trajectory starting from a fixed direction is stable if and only if its convergence rate is strictly less than one, equivalently $\gamma_{F}\left(\theta_{1}^{*}\right)<1$. A simple expression of $\gamma_{F}\left(\theta_{1}^{*}\right)$ is given below.

Lemma 4 ([13, Lemma 8 and Corollary 1]). Given BPLS defined by Eqs. (1) and (4), a fixed direction $V_{1}\left(\theta_{1}^{*}\right)$ is stable if and only if its convergence rate $\gamma_{F}\left(\theta_{1}^{*}\right)<1$. Furthermore if $\theta_{1}^{*} \neq 0$, then

$\gamma_{F}\left(\theta_{1}^{*}, \theta_{2}^{*}\right)=\frac{\exp \left(\lambda_{1} \tau_{1}+\lambda_{2} \tau_{2}\right)}{\frac{d \mathscr{F}_{1}}{d \theta_{1}} \frac{d \mathscr{F}_{2}}{d \theta_{2}}}$.

If a fixed direction $V_{1}\left(\theta_{1}^{*}\right)$ is attractive in an interval I of $\theta_{1}$, then in view of Definition 3, all the trajectories starting from a direction $V_{1}\left(\theta_{1}\right)$ where $\theta_{1}$ is in I, will eventually behave as a trajectory starting from $V_{1}\left(\theta_{1}^{*}\right)$. This observation leads to the following result.
Lemma 5 ([13, Theorem 1]). Let $V_{1}\left(\theta_{1}^{*}\right)$ be a fixed direction which is attractive on an interval I and consider a trajectory with initial condition $K_{1} V_{1}\left(\theta_{1}\right)$ where $K_{1}>0$ and $\theta_{1} \in I$. Then, the trajectory is stable (decays to origin as $t \rightarrow \infty$ ) if and only if the fixed direction $V_{1}\left(\theta_{1}^{*}\right)$ is stable.

Let $\mathcal{T}_{1}\left(\theta_{1}\right)=\theta_{11}$. Then, the ratio $\frac{\cot \theta_{1}+b_{1}}{\cot \theta_{11}+b_{1}}$ is important to determine the existence and the interval of attraction of a fixed direction. More precisely, if $\frac{\cot \theta_{1}+b_{1}}{\cot \theta_{11}+b_{1}}=1$, then $\theta_{1}=\theta_{11}$ which implies that $\theta_{1}$ is a fixed direction. On the other hand, if $\frac{\cot \theta_{1}+b_{1}}{\cot \theta_{11}+b_{1}}>1($ or $<1)$, then this information can be used to determine the interval of attraction. This is summarized by the following result in [13].

Lemma 6 ([13, Lemma 9]). Let $\theta_{1} \neq 0$ be in the domain of $\mathcal{T}_{1}\left(\theta_{1}\right)$. Then,

$\frac{\cot \theta_{1}+b_{1}}{\cot \theta_{11}+b_{1}}=\frac{d \mathcal{F}_{1}}{d \theta_{1}} \frac{d \mathcal{F}_{2}}{d \theta_{2}}$

where $\theta_{2}:=\mathcal{g}_{1}\left(\mathcal{F}_{1}\left(\theta_{1}\right)\right)$ is a decreasing function of $\theta_{1}$ and the following hold.

(1) If $\theta_{1}>0$ and $\frac{\cot \theta_{1}+b_{1}}{\cot \theta_{11}+b_{1}}>1$, then $\theta_{1}<\theta_{11}$; if $\theta_{1}>0$ and $\frac{\cot \theta_{1}+b_{1}}{\cot \theta_{11}+b_{1}}<1$, then $\theta_{1}>\theta_{11}$.

(2) If $\theta_{1}<0$ and $\frac{\cot \theta_{1}+b_{1}}{\cot \theta_{11}+b_{1}}<1$, then $\theta_{1}<\theta_{11}$; if $\theta_{1}<0$ and $\frac{\cot \theta_{1}+b_{1}}{\cot \theta_{11}+b_{1}}>1$, then $\theta_{1}>\theta_{11}$.

\section{Main results}

Recall from the previous section that there are two different bases $\left\{\hat{\mathbf{x}}_{i}, \mathbf{y}_{i}, i=1,2\right\}$ of the separating plane $\mathscr{H}$. The main result of this paper shows that "the way these bases are placed on $\mathscr{H}$ with respect to each other" is crucial for GAS of BPLS. In order to make this more concrete, let us recall the coupling constant $\mathscr{B}_{1}$ given in Eq. (15).

$\mathcal{B}_{1}:=\frac{a_{11}-\lambda_{1}+a_{21} \lambda_{2}}{a_{21} w_{2}\left(1+b_{2}^{2}\right)}$.

In view of Eqs. (7) and (8) the tangent of the angle between $\mathbf{y}_{1}$ and $V_{1}\left(-\phi_{1}\right)$ on $\mathcal{H}$ is $\frac{a_{21}}{a_{11}-\lambda_{1}}$. Similarly, the tangent of the angle between $\mathbf{y}_{2}$ and $V_{2}\left(-\phi_{2}\right)$ is $-1 / \lambda_{2}$. If $\mathscr{B}_{1}>0$, then $a_{11}-\lambda_{1}+$ $a_{21} \lambda_{2}>0$ and $\frac{a_{21}}{a_{11}-\lambda_{1}}<-\frac{1}{\lambda_{2}}$. Consequently, $-\mathbf{y}_{1}$ is in the cone bordered by $V_{2}\left(-\phi_{2}\right)$ and $\mathbf{y}_{2}$. On the other hand, if $\mathscr{B}_{1}<0$, then $a_{11}-\lambda_{1}+a_{21} \lambda_{2}<0$ and this implies that $-\mathbf{y}_{1}$ is outside the cone bordered by $V_{2}\left(-\phi_{2}\right)$ and $\mathbf{y}_{2}$. Thus, $\mathscr{B}_{1}$ determines how the modes are coupled on $\mathcal{H}$.

Observe that if $\mathbf{y}_{1}$ and $\mathbf{y}_{2}$ are on the same line then $\mathscr{B}_{1}=0$. This configuration yields the simplest geometry of BPLS in $\mathbb{R}^{3}$, because the planes spanned by $\left\{\hat{\mathbf{x}}_{i}, \mathbf{y}_{i}, i=1,2\right\}$ intersect in a line on $\operatorname{ker} \mathbf{c}^{T}(=\mathscr{H})$. This case is investigated in [13] for every possible combination of the eigenvalues of the two modes of BPLS as mentioned in the Introduction. In the rest of the paper we assume that the coupling constant $\mathscr{B}_{1}>0$. Therefore, the geometry being considered and the results obtained in this paper are radically different from [13]. The geometry for the case $\mathscr{B}_{1}>0$ is depicted in Fig. 4.

It is mentioned in the previous section that the ratio $\frac{\cot \theta_{1}+b_{1}}{\cot \theta_{11}+b_{1}}$ is important in determining the existence and the interval of attraction for fixed directions. This is also true for the case being considered in this paper, however the expression for the ratio is different from what is given by Lemma 6 . In order to see this difference let us first define $L_{1}$ and $L_{2}$.

$L_{1}:=\left[1-\mathscr{B}_{2}\left(\cot \left(\theta_{1}+w_{1} \tau_{1}\right)+b_{1}\right)\right]$,

$L_{2}:=\left[1+\mathscr{B}_{1}\left(\cot \left(\theta_{2}+w_{2} \tau_{2}\right)+b_{2}\right)\right]$. 


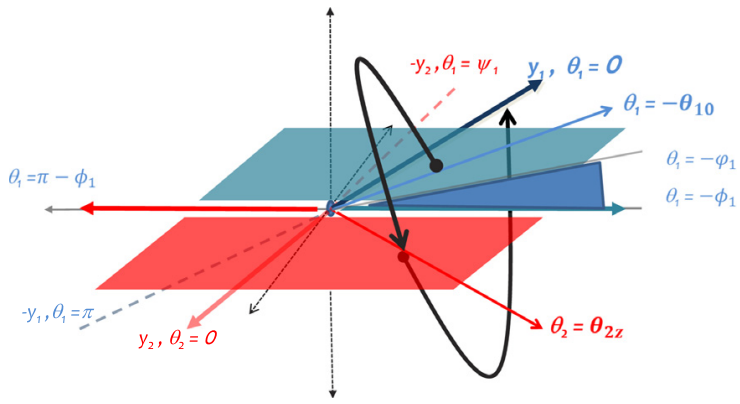

Fig. 5. Geometry of the directions given in Lemma 8(1).

Lemma 7. Let $\theta_{1} \neq 0$ be in the domain of $\mathcal{T}_{1}\left(\theta_{1}\right)$. Then,

$\frac{\cot \theta_{1}+b_{1}}{\cot \theta_{11}+b_{1}}=\frac{d \mathcal{F}_{1}}{d \theta_{1}} \frac{d \mathcal{F}_{2}}{d \theta_{2}} L_{1} L_{2}$.

\section{Proof (In the Appendix).}

In what follows, we show that under the assumptions $\sigma_{1}-\lambda_{1} \leq$ $0, \sigma_{2}-\lambda_{2}>0$, both $\frac{d \mathscr{F}_{1}}{d \theta_{1}} \frac{d \mathscr{F}_{2}}{d \theta_{2}}$ and $L_{1} L_{2}$ become a decreasing function of $\theta_{1}$ for the entire domain of $\tau_{1}$. This enables us to find the fixed directions and the intervals where they are attractive easily. However, for the other combinations of signs of $\sigma_{i}-\lambda_{i}$, this is not an easy task and the main problem is to prove the uniqueness of fixed directions in a certain interval of $\theta_{1}$.

Lemma 8. Suppose that $\mathscr{B}_{1}>0, \sigma_{1}-\lambda_{1} \leq 0$, and $\sigma_{2}-\lambda_{2}>0$. Then, the following hold.

(1) There exist $-\theta_{10}<-\theta_{1 z}<0$ and $0<\theta_{2 z}$ such that $\theta_{2 z}=g_{1}$ $\left(\mathcal{F}_{1}\left(-\theta_{10}\right)\right), g_{1}\left(\mathcal{F}_{1}\left(-\theta_{1 z}\right)\right)=0, \mathcal{T}_{1}\left(-\theta_{10}\right)=0$ and $\mathcal{T}_{1}\left(-\theta_{1 z}\right)$ $=\psi_{1}$.

(2) If $\theta_{1}$ is in the domain of $\mathcal{T}_{1}(\cdot)$ and $\theta_{1}>0$ or if $\theta_{1} \in\left(-\varphi_{1},-\theta_{10}\right]$, then $L_{1} L_{2}$ is a decreasing function of $\theta_{1}$ and

$$
\lim _{\theta_{1} \rightarrow\left(\pi-\phi_{1}\right)^{-}} L_{1} L_{2}>1, \quad \lim _{\theta_{1} \rightarrow-\varphi_{1}^{+}} L_{1} L_{2}=1 .
$$

(3) If $\theta_{1}$ is in the domain of $\mathcal{T}_{1}(\cdot)$, then $\theta_{1}<\mathcal{T}_{1}\left(\theta_{1}\right)$ over the interval $\left[-\theta_{10}, \psi_{1}\right]$ and $\lim _{\theta_{1} \rightarrow 0^{ \pm}} L_{1} L_{2}= \pm \infty$.

(4) Over the interval $\left(-\varphi_{1}, \pi-\phi_{1}\right], \frac{d \mathcal{F}_{1}}{d \theta_{1}} \frac{d \mathcal{F}_{2}}{d \theta_{2}}$ is a strictly decreasing function of $\theta_{1}$ and if $\sigma_{1}-\lambda_{1}<0$, then at the end points of the domain of $\mathcal{T}_{1}(\cdot)$ we get

$$
\left.\frac{d \mathcal{F}_{1}}{d \theta_{1}} \frac{d \mathcal{F}_{2}}{d \theta_{2}}\right|_{\theta_{1}=\pi-\phi_{1}}=0 \text { and } \lim _{\theta_{1} \rightarrow-\varphi_{1}^{+}} \frac{d \mathcal{F}_{1}}{d \theta_{1}} \frac{d \mathcal{F}_{2}}{d \theta_{2}}=\infty .
$$

(5) If $\sigma_{1}-\lambda_{1}=0$, then it follows that

$$
\lim _{\theta_{1} \rightarrow-\varphi_{1}^{+}} \frac{d \mathcal{F}_{1}}{d \theta_{1}} \frac{d \mathcal{F}_{2}}{d \theta_{2}}=1
$$

\section{Proof (In the Appendix).}

The facts that $\mathcal{T}_{1}\left(-\theta_{10}\right)=0$ and $\mathcal{T}_{1}\left(-\theta_{1 z}\right)=\psi_{1}$ are demonstrated in figures 5 and 6 .

We now present the main result of this paper.

Theorem 1. Consider BPLS given by Eqs. (1) and (4) with Assumptions A1-A3. Suppose that $\mathscr{B}_{1}>0, \sigma_{1}-\lambda_{1} \leq 0$, and $\sigma_{2}-\lambda_{2}>0$. Then, the following hold.

(1) There exist two pairs of unique fixed directions $V\left(\theta_{1}^{* 1},-\theta_{2}^{* 1}\right)$ and $V\left(-\theta_{1}^{* 2}, \theta_{2}^{* 2}\right)$, where $\theta_{1}^{* 1} \in\left[0, \pi-\phi_{1}\right]$, and $-\theta_{2}^{* 1} \in$ $\left[-\phi_{2},-\psi_{2}\right]$ and $-\theta_{1}^{* 2} \in\left(-\varphi_{1},-\theta_{10}\right), \theta_{2}^{* 2} \in\left[0, \pi-\phi_{2}\right)$.

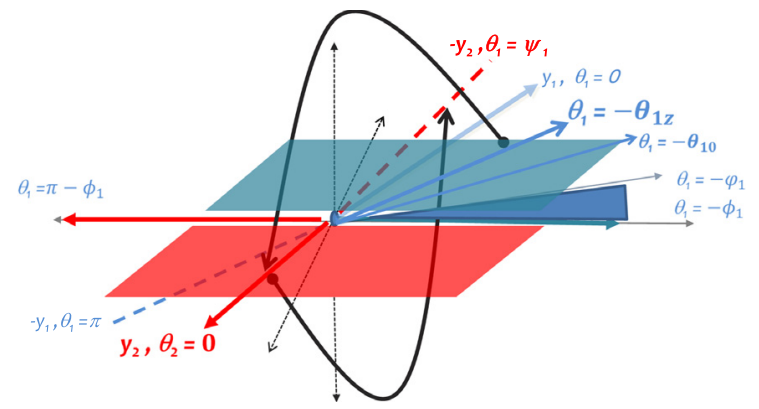

Fig. 6. Geometry of the directions given in Lemma 8(1).

(2) BPLS is GAS if and only if $\lambda_{i}<0$ for $i=1,2$ and the trajectories starting from the pair of fixed directions $V\left(\theta_{1}^{* 1},-\theta_{2}^{* 1}\right)$ are stable or equivalently $\gamma_{F}^{*}\left(\theta_{1}^{* 1},-\theta_{2}^{* 1}\right)<1$.

Proof. Since $\sigma_{1}-\lambda_{1} \leq 0$, Lemma 1(2)(a) implies that the trajectories starting from $V_{1}\left(\theta_{1}\right)$, where $\theta_{1} \in\left[-\phi_{1},-\varphi_{1}\right]$, are nontransitive and decay to the origin if and only if $\lambda_{1}<0$. If $\theta_{1}>0$, then by Lemma 8(2), (4), (5) it follows that $\frac{d \mathcal{F}_{1}}{d \theta_{1}} \frac{d \mathcal{F}_{2}}{d \theta_{2}} L_{1} L_{2}$ is decreasing from $\infty$ at $\theta_{1}=0$ to zero at $\theta_{1}=\pi-\phi_{1}$. Thus, there exists a unique $\theta_{1}^{* 1} \in\left[0, \pi-\phi_{1}\right]$ such that $\frac{\cot \theta_{1}+b_{1}}{\cot \theta_{11}+b_{1}}=1$ at $\theta_{1}=\theta_{1}^{* 1}$. Observe that $\frac{\cot \theta_{1}+b_{1}}{\cot \theta_{11}+b_{1}}>1$ if $\theta_{1}<\theta_{1}^{* 1}$, and $\frac{\cot \theta_{1}+b_{1}}{\cot \theta_{11}+b_{1}}<1$ if $\theta_{1}>\theta_{1}^{* 1}$. Consequently, Lemma 6(1) implies that the fixed direction $V\left(\theta_{1}^{* 1},-\theta_{2}^{* 1}\right)$, where $-\theta_{2}^{* 1}=g_{1}\left(\mathcal{F}_{1}\left(\theta_{1}^{* 1}\right)\right)$, is attractive in the interval $\left[0, \pi-\phi_{1}\right]$. Similarly, if $\theta_{1} \in\left(-\varphi_{1},-\theta_{10}\right]$ and $\sigma_{1}-\lambda_{1}<0$, then Lemma 8(2), (3) and (4) implies that $\frac{d \mathcal{F}_{1}}{d \theta_{1}} \frac{d \mathcal{F}_{2}}{d \theta_{2}} L_{1} L_{2}$ is strictly decreasing from $\infty$ at the limit $\theta_{1}=-\varphi_{1}$ to zero at $\theta_{1}=-\theta_{10}$. Thus, there exists a unique $-\theta_{1}^{* 2} \in\left(-\varphi_{1},-\theta_{10}\right]$ such that $\frac{\cot \theta_{1}+b_{1}}{\cot \theta_{11}+b_{1}}=1$ at $\theta_{1}=-\theta_{1}^{* 2}$. Since $\frac{\cot \theta_{1}+b_{1}}{\cot \theta_{11}+b_{1}}>1$ if $\theta_{1}<$ $-\theta_{1}^{* 2}$ and $\frac{\cot \theta_{1}+b_{1}}{\cot \theta_{11}+b_{1}}<1$ if $\theta_{1}>-\theta_{1}^{* 2}$, Lemma 6(2) implies that the fixed direction $V\left(-\theta_{1}^{* 2}, \theta_{2}^{* 2}\right)$, where $\theta_{2}^{* 2}=g_{1}\left(\mathcal{F}_{1}\left(-\theta_{1}^{* 2}\right)\right)$, is repulsive. On the other hand, if $\theta_{1} \in\left[-\theta_{10}, 0\right)$, by Lemma 8(3) we have $L_{1} L_{2} \leq 0$ where the equality holds at $\theta_{1}=-\theta_{10}$. Consequently, $\frac{\cot \theta_{1}+b_{1}}{\cot \theta_{11}+b_{1}}<0$ which implies, by Lemma 6(2), that $\mathcal{T}_{1}\left(\theta_{1}\right)=\theta_{11}>0$ for any $\theta_{1} \in\left[-\theta_{10}, 0\right)$. Hence, $V\left(\theta_{1}^{* 1},-\theta_{2}^{* 1}\right)$ is attractive in the interval $\left(-\theta_{1}^{* 2}, \pi-\phi_{1}\right]$ and the invariant cone $\mathcal{C}_{1}$ is attractive in the interval $\left[-\phi_{1},-\theta_{1}^{* 2}\right)$. Furthermore, note that since $\lim _{\theta_{1} \rightarrow-\varphi_{1}^{+}} L_{1} L_{2}=1$ and $L_{1} L_{2}$ decreases as $\theta_{1}$ increases, it follows that $\left.\frac{d \mathcal{F}_{1}}{d \theta_{1}} \frac{d \mathcal{F}_{2}}{d \theta_{2}}\right|_{\theta_{1}=-\theta_{1}^{* 2}} \geq 1$. Then, Lemma 4 implies that $\gamma_{F}\left(-\theta_{1}^{* 2}, \theta_{2}^{* 2}\right)<1$ and consequently the repulsive fixed pair of directions $V\left(-\theta_{1}^{* 2}, \theta_{2}^{* 2}\right)$ is stable if and only if $\lambda_{1}$ is strictly negative. Convergence rate of $V\left(\theta_{1}^{* 1},-\theta_{2}^{* 1}\right)$ can be calculated by a simple algorithm outlined in Remark 6 .

On the other hand, if $\sigma_{1}-\lambda_{1}=0$, then Lemma 8(2), (4) and (5) implies that $\frac{d \mathscr{F}_{1}}{d \theta_{1}} \frac{d \mathcal{F}_{2}}{d \theta_{2}} L_{1} L_{2}$ is decreasing from 1 at the limit $\theta_{1}=-\varphi_{1}=-\frac{\pi}{2}$ to zero at $\theta_{1}=-\theta_{10}$. Thus, the fixed direction $V\left(-\theta_{1}^{* 2}, \theta_{2}^{* 2}\right)$ and $\mathcal{C}_{1}$ merge to $V_{1}\left(-\frac{\pi}{2}\right)$ and the fixed direction $V\left(\theta_{1}^{* 1},-\theta_{2}^{* 1}\right)$ becomes attractive in the interval $\left(-\frac{\pi}{2}, \frac{\pi}{2}\right]$ and $\mathcal{C}_{1}$ is repulsive. Consequently, for both cases $\left(\sigma_{1}-\lambda_{1}<0\right.$ and $\left.\sigma_{1}-\lambda_{1}=0\right)$, BPLS is GAS if and only if $\lambda_{i}<0$ for $i=1,2$ and the trajectories starting from $V\left(\theta_{1}^{* 1},-\theta_{2}^{* 1}\right)$ are stable or equivalently $\gamma_{F}\left(\theta_{1}^{* 1},-\theta_{2}^{* 1}\right)<1$.

The position of the fixed directions are shown in Fig. 7 where curved arrows indicate the points where the trajectories hit $\mathscr{H}$ and change mode for attractive and repulsive fixed directions.

Remark 6. In Example 1 given below we demonstrate that the coupling constant $\mathscr{B}_{1}$ can be changed without changing the 


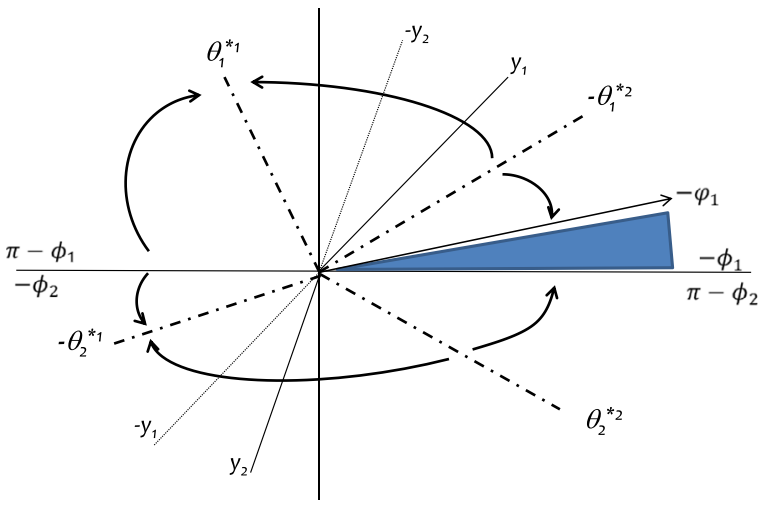

Fig. 7. Attractivity and repulsivity of fixed directions.

eigenvalues of subsystems and this change can make BPLS GAS or unstable. If the vector field is assumed to be continuous, then $a_{11}=0, a_{21}=1$ and the coupling constant $\mathscr{B}_{1}$ given in Eq. (15) can be written as follows.

$\mathcal{B}_{1}:=\frac{-\lambda_{1}+\lambda_{2}}{w_{2}\left(1+b_{2}^{2}\right)}$.

This implies that the coupling constant cannot be changed without changing the eigenvalues of subsystems. Thus, the effect of the coupling constant, as demonstrated by Example 1 given below, cannot be observed in BPLS with continuous vector fields. This constitutes the basic difference of our work with the works in the literature which are based on continuous vector fields. The coupling constants of BPLS given in Example 1 are directly calculated by using Eq. (14). For the calculation of the convergence rate an algorithm similar to the one given in [14, Remark 6] can be used. This algorithm starts with $\theta_{1}$, which is an angle in the interval of attraction of the fixed direction, and calculates $\theta_{11}:=\mathcal{T}_{1}\left(\theta_{1}\right)$, $\theta_{12}:=\mathcal{T}_{1}^{2}\left(\theta_{1}\right)$, up to $\theta_{1 k}:=\mathcal{T}_{1}^{k}\left(\theta_{1}\right)$ as described by Eqs. (22) and (23). For the sake of completeness, we give below a brief outline of the algorithm.

Algorithm. Let $\theta_{1}$ be an angle in the interval of attraction of the fixed direction and consider a trajectory starting along the direction $V_{1}\left(\theta_{1}\right)$ in $\mathscr{H} \cap \varsigma_{1}$.

- This trajectory hits $\mathscr{H}$ at $\theta_{1}+w_{1} \tau_{1}$ in $\mathscr{H} \cap \wp_{2}$. Calculate $\theta_{1}+w_{1} \tau_{1}$ numerically using Eq. (14).

- The new initial condition of the trajectory is along the direction $V_{2}\left(\theta_{2}\right)$ in $\mathscr{H} \cap \varsigma_{2}$. Substitute $\theta_{1}+w_{1} \tau_{1}$ in place of $\theta_{1}$ in Eq. (16) and calculate $\theta_{2}$.

- The trajectory continues in mode 2 and hits $\mathscr{H}$ at $\theta_{2}+w_{2} \tau_{2}$ in $\mathscr{H} \cap \varsigma_{1}$ again. Use Eq. (14) again with $\theta_{2}$ and calculate $\theta_{2}+w_{2} \tau_{2}$ numerically.

- At this point the new initial condition of the trajectory is along the direction $V_{1}\left(\theta_{11}\right)$ in $\mathscr{H} \cap \varsigma_{1}$. Substitute $\theta_{2}+w_{2} \tau_{2}$ in place of $\theta_{2}$ and $\mathcal{T}_{1}\left(\theta_{1}\right):=\theta_{11}$ instead of $\theta_{1}$ in Eq. (18) and calculate $\theta_{11}$.

- Repeat the steps above starting with $\theta_{11}$ to calculate $\theta_{12}$, then start with $\theta_{12}$ and calculate $\theta_{13}$, and continue until $\theta_{1 k}$ is sufficiently close to $\theta_{1, k-1}$ (a tolerance about $10^{-5}$ is enough). Then, take $\theta_{1 k}$ as the fixed direction.

- Use $\theta_{1 k}$ and Eq. (24) to calculate the convergence rate $\gamma_{F}$.

Example 1. Consider the following BPLS.

$\dot{\mathbf{x}}(t)=\left\{\begin{array}{ll}\mathbf{A}_{1} \mathbf{x}(t) & \text { if } \mathbf{c}^{T} \mathbf{x}(t) \geq 0 \\ \mathbf{A}_{2} \mathbf{x}(t) & \text { if } \mathbf{c}^{T} \mathbf{x}(t) \leq 0\end{array}\right\}$ where

$\mathbf{A}_{1}=\left[\begin{array}{ccc}\frac{1}{2} & -\frac{25}{4} & 0 \\ 1 & \frac{-5}{2} & 0 \\ 0 & 1 & -0.8\end{array}\right]$,

$\mathbf{A}_{2}=\left[\begin{array}{ccc}0 & 0 & -3.616 \\ 1 & 0 & -8.88 \\ 0 & 1 & 0\end{array}\right]$

Note that the spectrum of $\mathbf{A}_{1}$ is $\left\{\lambda_{1}, \sigma_{1} \mp j w_{1}\right\}=\{-0.8$, $-1 \mp 2 j\}$ and the spectrum of $\mathbf{A}_{2}$ is $\left\{\lambda_{2}, \sigma_{2} \mp j w_{2}\right\}=\{-0.4$, $0.2 \mp 3 j\}$.

This BPLS is GAS with convergence rate $\gamma_{F}=0.9120$ and coupling constant $B_{1}=0.2885$. The entries of the system matrix $\mathbf{A}_{1}$ can be changed without changing its spectrum as follows.

$\mathbf{A}_{1}=\left[\begin{array}{ccc}1 & -8 & 0 \\ 1 & -3 & 0 \\ 0 & 1 & -0.8\end{array}\right]$

Thus, $\mathbf{A}_{1}$ still have the same spectrum $\left\{\lambda_{1}, \sigma_{1} \mp j w_{1}\right\}=$ $\{-0.8,-1 \mp 2 j\}$. But BPLS is unstable and the convergence rate is $\gamma_{F}=1.2637$ and the coupling constant is $B_{1}=0.4487$.

\section{Conclusions and future works}

The main result of this paper provides a complete set of verifiable necessary and sufficient conditions for GAS of a class of BPLS in $\mathbb{R}^{3}$ under the assumptions (A1-A3) and $\sigma_{1}-\lambda_{1} \leq 0, \sigma_{2}-\lambda_{2}>0$. It is shown that the trajectories which change mode infinite number of times as $t \rightarrow \infty$ eventually converge to the trajectories which start from the fixed directions on $\mathcal{H}$. Consequently, GAS is determined by the stability of the trajectories starting from the fixed directions. Thus, the existence of trajectories which change mode infinite number of times (as $t \rightarrow \infty$ ) is crucial in BPLS. It is also shown by Example 1 that the coupling constant can be changed without changing the eigenvalues of the subsystem and this change can make BPLS unstable or GAS. This means that the stabilizability of BPLS is both an eigenvalue and eigenvector assignment problem. Thus, stabilizability of BPLS can be considered as a natural future work. Moreover, a complete set of verifiable necessary and sufficient conditions for GAS of all classes of BPLS $\mathbb{R}^{3}$ with all possible combinations of eigenvalues and geometric configurations is still a legitimate future work.

\section{Acknowledgment}

This work is supported in part by 2219 programme of the Scientific and Technological Research Council of Turkey, TUBITAK.

\section{Appendix}

Proof of Lemma 7. Suppose that a trajectory starts from $\mathscr{H} \cap \mho_{1}$ along the direction $V_{1}\left(\theta_{1}\right)$ where $\theta_{1}$ is in the domain of $\mathcal{T}_{1}$ and changes mode along the direction $V_{1}\left(\theta_{1}+w_{1} \tau_{1}\right)$. Then, in view of Eq. (16) there exists $\theta_{2}$ such that

$$
\cot \left(\theta_{2}\right)+b_{2}=\frac{\cot \left(\theta_{1}+w_{1} \tau_{1}\right)+b_{1}}{\mathcal{C}_{1}-\mathcal{B}_{1}\left(\cot \left(\theta_{1}+w_{1} \tau_{1}\right)+b_{1}\right)} .
$$

Similarly, the trajectory continues in $\varsigma_{2}$ and changes mode along the direction $V_{2}\left(\theta_{2}+w_{2} \tau_{2}\right)$. Thus, in view of Eq. (18) there exists $\theta_{11}$ such that

$\cot \left(\theta_{11}\right)+b_{1}=\frac{\cot \left(\theta_{2}+w_{2} \tau_{2}\right)+b_{2}}{\mathcal{C}_{2}+\mathscr{B}_{2}\left(\cot \left(\theta_{2}+w_{2} \tau_{2}\right)+b_{2}\right)}$. 
Also recall from Lemma 2(2) that

$\frac{d \mathcal{F}_{1}}{d \theta_{1}} \frac{d \mathcal{F}_{2}}{d \theta_{2}}=\frac{d\left(\theta_{1}+w_{1} \tau_{1}\right)}{d \theta_{1}} \frac{d\left(\theta_{2}+w_{2} \tau_{2}\right)}{d \theta_{2}}$.

Since, $\mathcal{C}_{1} \mathcal{C}_{2}=1, \mathcal{C}_{1} \mathscr{B}_{2}=\mathscr{B}_{1}$, and $\mathcal{C}_{2} \mathscr{B}_{1}=\mathscr{B}_{2}$, combining the equations above, we get

$\frac{\cot \theta_{1}+b_{1}}{\cot \theta_{11}+b_{1}}=\frac{d \mathcal{F}_{1}}{d \theta_{1}} \frac{d \mathcal{F}_{2}}{d \theta_{2}} L_{1} L_{2}$.

Proof of Lemma 8. (1) Suppose that $\mathscr{B}_{1}>0$. Then $\mathscr{g}_{2}\left(\mathcal{F}_{2}().\right)$ maps $\left[0, \pi-\phi_{2}\right]$ onto $\left[-\phi_{1}, \psi_{1}\right]$. Since $g_{2}\left(\mathcal{F}_{2}().\right)$ is a continuous function and $\psi_{1}>0$, the existence of $\theta_{2 z}>0$ such that $g_{2}\left(\mathcal{F}_{2}\left(\theta_{2 z}\right)\right)=0$ is clear. Since $\sigma_{1}-\lambda_{1} \leq 0, g_{1}\left(\mathcal{F}_{1}().\right)$ maps $\left(-\varphi_{1}, 0\right]$ onto $\left[-\psi_{2}, \pi-\phi_{2}\right)$. Since $g_{1}\left(\mathcal{F}_{1}().\right)$ is a continuous function there exists of $\theta_{10}>0$ such that $g_{1}\left(\mathcal{F}_{1}\left(-\theta_{10}\right)\right)=\theta_{2 z}>0$. Note that $\frac{d \mathcal{F}_{2}}{d \theta_{2}} \leq 0$ by Lemma $2(2)$ and by Eq. (16) we have

$$
\begin{aligned}
& \frac{d \theta_{2}}{d\left(\theta_{1}+w_{1} \tau_{1}\right)} \\
& =\frac{C_{1} \sin ^{2} \theta_{2}}{\sin ^{2}\left(\theta_{1}+w_{1} \tau_{1}\right)\left[C_{1}-B_{1}\left(\cot \left(\theta_{1}+w_{1} \tau_{1}\right)+b_{1}\right)\right]^{2}}>0,
\end{aligned}
$$

and

$\frac{d \theta_{2}}{d \theta_{1}}=\frac{d \theta_{2}}{d\left(\theta_{1}+w_{1} \tau_{1}\right)} \frac{d \mathcal{F}_{1}}{d \theta_{1}}$.

Thus, it follows that $\theta_{2}$ decreases as $\theta_{1}$ increases. This implies the existence of $\theta_{1 z}<\theta_{10}$ such that $g_{1}\left(\mathcal{F}_{1}\left(-\theta_{1 z}\right)\right)=0$. Finally, in view of the definition of $\mathcal{T}_{1}($.$) , it is clear that \mathcal{T}_{1}\left(-\theta_{1 z}\right)=\psi_{1}$ and $\mathcal{T}_{1}\left(-\theta_{10}\right)=0$.

(2) Since $L_{1}=\left[1-\mathscr{B}_{2}\left(\cot \left(\theta_{1}+w_{1} \tau_{1}\right)+b_{1}\right)\right]$, it follows that

$\frac{d L_{1}}{d \theta_{1}}=\frac{\mathcal{B}_{2}}{\sin ^{2}\left(\theta_{1}+w_{1} \tau_{1}\right)} \frac{d \mathcal{F}_{1}}{d \theta_{1}}$.

Since $\frac{d \mathscr{F}_{1}}{d \theta_{1}} \leq 0$ by Lemma $2(2)$ and $\mathscr{B}_{2}>0, L_{1}$ is a decreasing function of $\theta_{1}$ over the entire domain of $\mathcal{T}_{1}(\cdot)$. Similarly, since $L_{2}=\left[1+\mathscr{B}_{1}\left(\cot \left(\theta_{2}+w_{2} \tau_{2}\right)+b_{2}\right)\right]$, it also follows that

$\frac{d L_{2}}{d \theta_{1}}=\frac{-\mathscr{B}_{1}}{\sin ^{2}\left(\theta_{2}+w_{2} \tau_{2}\right)} \frac{d \mathcal{F}_{2}}{d \theta_{2}} \frac{d \theta_{2}}{d \theta_{1}}$.

Then, again in view of Lemma 2(2) we have $\frac{d L_{2}}{d \theta_{1}} \leq 0$. Consequently, $L_{2}$ is also a decreasing function of $\theta_{1}$ over the entire domain $\left(-\varphi_{1}, \pi-\phi_{1}\right]$ of $\mathcal{T}_{1}(\cdot)$.

(3) If $\theta_{1} \in\left(-\varphi_{1},-\theta_{10}\right]$, then since $\pi \leq \theta_{1}+w_{1} \tau_{1} \leq 2 \pi-\phi_{1}$ by Remark 3 , it follows that $\left(\cot \left(\theta_{1}+w_{1} \tau_{1}\right)+b_{1}\right)>0$ and $0 \leq$ $L_{1} \leq 1$ as $\theta_{1}+w_{1} \tau_{1}>\pi+\psi_{1}$. Similarly, since $g_{1}\left(\mathcal{F}_{1}\left(-\theta_{10}\right)\right)=$ $\theta_{2 z}>0$, it follows that $\left(\cot \left(\theta_{2}+w_{2} \tau_{2}\right)+b_{2}\right)<0$ and $0 \leq L_{2} \leq 1$ as $\cot \left(\theta_{2}+w_{2} \tau_{2}\right) \geq-\cot \psi_{2}$. Consequently, we get $L_{1} \geq 0, L_{2} \geq 0$ and $L_{1} L_{2}$ is a decreasing function of $\theta_{1}$ if $\theta_{1} \in\left(-\varphi_{1},-\theta_{10}\right]$. In fact, since $g_{1}\left(\mathcal{F}_{1}\left(-\theta_{10}\right)\right)=\theta_{2 z}$, it follows that $\cot \left(\theta_{2}+w_{2} \tau_{2}\right)=\cot \left(\pi-\psi_{2}\right)$ at $\theta_{1}=-\theta_{10}$ and $L_{2}\left(\pi-\psi_{2}\right)=0$. This implies that $L_{1} L_{2}=0$ at $\theta_{1}=-\theta_{10}$.

On the other hand, if $\theta_{1}>0$, then since $\pi-\phi_{1}<\theta_{1}+$ $w_{1} \tau_{1} \leq \pi$ by Remark 1 , we have $\left(\cot \left(\theta_{1}+w_{1} \tau_{1}\right)+b_{1}\right) \leq 0$. Since $g_{1}\left(\mathcal{F}_{1}\left(\theta_{1}\right)\right) \in\left(-\phi_{2},-\psi_{2}\right]$, it also follows that $\pi \leq$ $\theta_{2}+w_{2} \tau_{2}<\pi+\phi_{2}$ by Remark 3, which implies that $\left(\cot \left(\theta_{2}+w_{2} \tau_{2}\right)+b_{2}\right) \geq 0$. Consequently, $L_{1} \geq 1, L_{2} \geq 1$ and $L_{1} L_{2}$ is a decreasing function of $\theta_{1}$ if $\theta_{1}>0$.

At $\theta_{1}=\pi-\phi_{1}$, we have $\cot \left(\theta_{1}+w_{1} \tau_{1}\right)+b_{1}=0$ which implies that $L_{1}=1$ and $\cot \left(\theta_{2}+w_{2} \tau_{2}\right)+b_{2}>0$ as $\pi<$ $\theta_{2}+w_{2} \tau_{2}<\pi+\phi_{2}$. Consequently, we get $\left.L_{1} L_{2}\right|_{\theta_{1}=\pi-\phi_{1}}>1$. For the other end of the interval, we have

$\lim _{\theta_{1} \rightarrow-\varphi_{1}^{+}}\left(\mathcal{F}_{1}\left(\theta_{1}\right)\right)=2 \pi-\phi_{1} \Longrightarrow \lim _{\theta_{1} \rightarrow-\varphi_{1}^{+}} L_{1}=1$.
Since $V_{1}\left(2 \pi-\phi_{1}\right) \simeq V_{2}\left(\pi-\phi_{2}\right)$, this also implies that $\lim _{\theta_{1} \rightarrow-\varphi_{1}^{+}} L_{2}=1$. Consequently, we have $\lim _{\theta_{1} \rightarrow-\varphi_{1}^{+}} L_{1} L_{2}$ $=1$.

(4) If $\theta_{1} \in\left[-\theta_{10},-\theta_{1 z}\right]$, then $g_{1}\left(\mathcal{F}_{1}\left(\theta_{1}\right)\right) \in\left[0, \theta_{2 z}\right]$, which implies that $\cot \left(\theta_{2}+w_{2} \tau_{2}\right) \leq-\cot \psi_{2}$. Thus, $L_{2}<0$. Since $\theta_{1}+$ $w_{1} \tau_{1} \geq \pi+\psi_{1}$ it follows that $L_{1} \geq 0$. Consequently, $L_{1} L_{2}<0$. In fact, since $g_{1}\left(\mathcal{F}_{1}\left(-\theta_{1 z}\right)\right):=0$, it turns out that $\mathscr{F}_{1}\left(-\theta_{1 z}\right)=$ $\pi+\psi_{1}$ and this implies that $L_{1}=0$ at $\theta_{1}=-\theta_{1 z}$. However, since $V_{1}\left(\pi+\psi_{1}\right) \simeq V_{2}(0)$, we have $\theta_{2}+w_{2} \tau_{2}=\pi$. This implies that

$\lim _{\theta_{1}+w_{1} \tau_{1} \rightarrow \pi+\psi_{1}} L_{2}=-\infty$ and $\lim _{\theta_{1}+w_{1} \tau_{1} \rightarrow \pi+\psi_{1}} L_{1}=0$.

Using L'Hospital's Rule, the limit of $L_{1} L_{2}$ as $\theta_{1}+w_{1} \tau_{1} \rightarrow$ $\pi+\psi_{1}$ can be calculated easily as follows.

$$
\begin{aligned}
& \lim _{\theta_{1}+w_{1} \tau_{1} \rightarrow \pi+\psi_{1}} L_{1} L_{2} \\
= & \lim _{\theta_{1}+w_{1} \tau_{1} \rightarrow \pi+\psi_{1}} \frac{\left[1-\mathcal{B}_{2}\left(\left(\cot \left(\theta_{1}+w_{1} \tau_{1}\right)+b_{1}\right)\right)\right]}{\sin \left(\theta_{2}+w_{2} \tau_{2}\right)} \\
& \times\left[\sin \left(\theta_{2}+w_{2} \tau_{2}\right)\left(1+\mathscr{B}_{1} b_{2}\right)+\mathscr{B}_{1} \cos \left(\theta_{2}+w_{2} \tau_{2}\right)\right] \\
= & \lim _{\theta_{1}+w_{1} \tau_{1} \rightarrow \pi+\psi_{1}} \\
& \times \frac{\mathscr{B}_{2}}{\sin ^{2}\left(\theta_{1}+w_{1} \tau_{1}\right) \cos \left(\theta_{2}+w_{2} \tau_{2}\right) \frac{d \mathcal{F}_{2}}{d \theta_{2}} \frac{d \theta_{2}}{d\left(\theta_{1}+w_{1} \tau_{1}\right)}}\left[-\mathscr{B}_{1}\right]
\end{aligned}
$$

which is a finite negative number as $\cos \left(\theta_{2}+w_{2} \tau_{2}\right) \frac{d \mathcal{F}_{2}}{d \theta_{2}}$ and $\frac{d \theta_{2}}{d\left(\theta_{1}+w_{1} \tau_{1}\right)}$ are both $>0$. Thus, $L_{1} L_{2}<0$ if $\theta_{1} \in\left(-\theta_{10},-\theta_{1 z}\right]$. Consequently, $\frac{d \mathcal{F}_{1}}{d \theta_{1}} \frac{d \tilde{F}_{2}}{d \theta_{2}} L_{1} L_{2}<1$ and Lemma 6(2) implies that $\theta_{1}<\mathcal{T}_{1}\left(\theta_{1}\right)$.

Similarly, if $\theta_{1} \in\left(-\theta_{1 z}, 0\right]$, since $\theta_{1}+w_{1} \tau_{1}$ is a decreasing function of $\theta_{1}$ (Lemma 2(1)), we have $\theta_{1}+w_{1} \tau_{1}<\pi+$ $\psi_{1}$. This implies that $L_{1}<0$. On the other hand, since $g_{1}\left(\mathcal{F}_{1}\left(-\theta_{1 z}\right)\right)=0$ and $\theta_{2}+w_{2} \tau_{2}$ is an increasing function of $\theta_{1}$, it follows that $\left(\cot \left(\theta_{2}+w_{2} \tau_{2}\right)+b_{2}\right)>0$. Thus, $L_{2}>0$. Consequently, $L_{1} L_{2}<0$ again. Thus, we have $\theta_{1}<\mathcal{T}_{1}\left(\theta_{1}\right)$. In fact, $\lim _{\theta_{1} \rightarrow 0^{-}} L_{1}=-\infty$ as $\lim _{\theta_{1} \rightarrow 0^{-}} \theta_{1}+w_{1} \tau_{1}=\pi$. This implies that

$\lim _{\theta_{1} \rightarrow 0^{-}} L_{1} L_{2}=-\infty$.

On the other hand, $\lim _{\theta_{1} \rightarrow 0^{+}} L_{1}=\infty$ as $\lim _{\theta_{1} \rightarrow 0^{+}} \theta_{1}+$ $w_{1} \tau_{1}=\pi$. Thus, we also have

$\lim _{\theta_{1} \rightarrow 0^{+}} L_{1} L_{2}=\infty$.

The domain of $\mathcal{T}_{1}($.$) is \left(-\varphi_{1}, \pi-\phi_{1}\right]$. Note that the item 2.b of this lemma shows that $L_{1} L_{2} \leq 0$ over the interval $\left[-\theta_{10}, 0\right)$. Since $\frac{d \mathcal{F}_{1}}{d \theta_{1}} \frac{d \mathcal{F}_{2}}{d \theta_{2}} \geq 0$ over the entire domain of $\mathcal{T}_{1}($.), Lemma 6 implies that

$$
\frac{\cot \left(\theta_{1}\right)+b_{1}}{\cot \left(\theta_{11}\right)+b_{1}}<0
$$

Since $\theta_{1}<0$, we have $\cot \left(\theta_{1}\right)+b_{1}<0$. Consequently, $\cot \left(\theta_{11}\right)+b_{1}>0$ which implies that $\theta_{11}=\mathcal{T}_{1}\left(\theta_{1}\right)>\theta_{1}$. If $\theta_{1} \in\left[0, \psi_{1}\right]$, then $\mathcal{g}_{1}\left(\mathcal{F}_{1}(\cdot)\right)$ maps $\left[0, \psi_{1}\right]$ into $\left[-\phi_{2},-\psi_{2}\right]$. Since for any $\theta_{2} \in\left[-\phi_{2},-\psi_{2}\right]$, we have $\theta_{2}+w_{2} \tau_{2}>\pi$, it follows that $g_{2}\left(\mathcal{F}_{2}(\cdot)\right)$ maps $\left[-\phi_{2},-\psi_{2}\right]$ into $\left(\psi_{1}, \pi-\phi_{1}\right)$. Consequently, $\theta_{11}=\mathcal{T}_{1}\left(\theta_{1}\right)>\theta_{1}$ for any $\theta_{1} \in\left[-\theta_{10}, \psi_{1}\right]$.

If $\sigma_{1}-\lambda_{1}<0$ and $\sigma_{2}-\lambda_{2}>0$, then by Lemma 2(3), we get $\left.\frac{d \mathcal{F}_{2}}{d \theta_{2}}\right|_{\theta_{2}=-\phi_{2}}=0$ and $\lim _{\theta_{1} \rightarrow \pi-\phi_{1}} \frac{d \mathcal{F}_{1}}{d \theta_{1}}=-1$. Since $V_{1}\left(\pi-\phi_{1}\right) \simeq V_{2}\left(-\phi_{2}\right)$, it follows that $\left.\frac{d \mathcal{F}_{1}}{d \theta_{1}} \frac{d \mathcal{F}_{2}}{d \theta_{2}}\right|_{\theta_{1}=\pi-\phi_{1}}=0$. 
Since $\sigma_{1}-\lambda_{1}<0, \lim _{\theta_{1} \rightarrow-\varphi_{1}^{+}} \frac{d \mathscr{F}_{1}}{d \theta_{1}}=-\infty$ by Lemma 2(2). Thus, as $\theta_{1}$ increases over the interval $\left(-\varphi_{1}, \pi-\phi_{1}\right), \frac{d \mathcal{F}_{1}}{d \theta_{1}}$ increases from $-\infty$ to -1 or equivalently $\left|\frac{d \mathcal{F}_{1}}{d \theta_{1}}\right|$ decreases from $\infty$ to 1 . Furthermore, $\theta_{2}$ decreases from $\pi-\phi_{2}$ to $-\phi_{2}$ and Lemma 2(2) implies that $\frac{d \mathcal{F}_{2}}{d \theta_{2}}$ increases from -1 to zero or equivalently $\left|\frac{d \mathcal{F}_{2}}{d \theta_{2}}\right|$ decreases from 1 to zero. Consequently, $\frac{d \mathcal{F}_{1}}{d \theta_{1}} \frac{d \mathcal{F}_{2}}{d \theta_{2}}$ decreases from $\infty$ to zero. Consequently, $\frac{d \mathcal{F}_{1}}{d \theta_{1}} \frac{d \mathcal{F}_{2}}{d \theta_{2}}$ is a decreasing function of $\theta_{1}$ over the entire domain of $\mathcal{T}_{1}(\cdot)$ and

$\lim _{\theta_{1} \rightarrow-\varphi_{1}^{+}} \frac{d \mathcal{F}_{1}}{d \theta_{1}} \frac{d \mathcal{F}_{2}}{d \theta_{2}}=\infty$,

if $\sigma_{1}-\lambda_{1}<0$.

(5) If $\sigma_{1}-\lambda_{1}=0$, then by Lemma 2(2)(c) we have $\frac{d \mathcal{F}_{1}}{d \theta_{1}}=-1$ for all $\theta_{1}$ in the domain of $\mathcal{T}_{1}(\cdot)$. Hence, we get

$\lim _{\theta_{1} \rightarrow-\varphi_{1}^{+}} \frac{d \mathcal{F}_{1}}{d \theta_{1}} \frac{d \mathcal{F}_{2}}{d \theta_{2}}=1$,

where $\varphi_{1}=\frac{\pi}{2}$.

\section{References}

[1] M. di Bernardo, C.J. Budd, A.R. Champneys, Kowalcyzk, Piecewise-Smooth Dynamical Systems, Springer-Verlag, 2008.

[2] A.F. Filippov, Differential Equations With Discontinuous Right Hand Sides, in: Mathematics and its Applications, Prentice-Hall, Dordrecht, The Netherlands, 1988.

[3] D. Liberzon, Switching in Systems and Control, Birkhauser, Boston, MA, 2003.

[4] A.J. Van der Schaft, J.M. Schumacher, An Introduction to Hybrid Dynamical Systems, in: Lecture Notes in Control and Information Sciences, vol. 251, Springer, Berlin, 2000.

[5] V. Utkin, Sliding Mode Control: Mathematical Tools, Design and Applications, Springer, 2011

[6] C. Georgescu, B. Brogliato, V. Acary, Switching, relay and complementarity systems: A tutorial on their well-posedness and relationships, Physica D 241 (2012) 1985-2002.
[7] Sun Zhendong, Stability of piecewise linear systems revisited, Annu. Rev. Control 34 (2) (2010) 221-223.

[8] M.K. Çamlıbel, Well-posed bimodal piecewise linear systems do not exhibit zeno behavior, in: Proc. of the 17th IFAC Congress Seoul Korea, 2008, pp. 7973-7978.

[9] J.I. Imura, A. van der Schaft, Characterization of well-posedness of piecewiselinear systems, IEEE Trans. Automat. Control 45 (9) (2000) 1600-1619.

[10] G. Sahan, V. Eldem, Well posedness conditions for bimodal piecewise affine systems, Systems Control Lett. 83 (2015) 9-18.

[11] L.Q. Thuan, M.K. Camlibel, On the existence, uniqueness and the nature of Carathéodory and Filippov solutions for bimodal piecewie affine sytems, Syst. Control Lett. 68 (2014) 76-85

[12] V. Eldem, G. Şahan, Stability of bimodal systems in $\mathbb{R}^{3}$, in: Proceedings of the Joint 48th IEEE Conference on Decision and Control and 28th Chinese Control Conference, ThA03.2, Shangay, China, December 16-18, 2009, pp. 3220-3225

[13] V. Eldem, G. Sahan, Structure and stability of bimodal systems in R3: Part I Appl. Comput. Math. Int. J. 13 (2) (2014) 206-229.

[14] V. Eldem, I. Oner, A note on the stability of bimodal systems in $\mathbb{R}^{3}$ with discontinuous vector fields, Internat. J. Control 88 (4) (2015) 729-744.

[15] Eren Yavuz, Jinglai Shen, Kanat Camlibel, Quadratic stability and stabilization of bimodal piecewise linear systems, Automatica 50 (5) (2014) 1444-1450.

[16] Jun-ichi Imura, Classification and stabilizability analysis of bimodal piecewise affine systems, Internat. J. Robust Nonlinear Control 12 (2002) 897-926.

[17] X. Liu, H. Lin, M. Chen Ben, Null controllability of planar bimodal piecewise linear systems, Internat. J. Control 84 (4) (2011) 766-782

[18] S. Huan, L. Qingdu, X.S. Yang, Chaos in three-dimensional hybrid systems and design of chaos generators, Nonlinear Dynam. 69 (2012) 1915-1927.

[19] Jun-ichi Imura, Kenji Kashima, Masami Kusano, Piecewise affine systems approach to control of biological networks, Phil. Trans. R. Soc. A 368 (1930) (2010) 4977-4993.

[20] V. Carmona, E. Freire, E. Ponce, F. Torres, Bifurcation of invariant cones in piecewise linear homogenous systems, Int. J. Bifurcation Chaos (IJBC) 15 (8) (2005) 2469-2484.

[21] V. Carmona, E. Freire, E. Ponce, F. Torres, The continuous matching of two stable linear systems can be unstable, Discrete Contin. Dynam. Systems 16 (3) (2006) 689-703.

[22] V. Carmona, E. Freire, Soledad Fernandez-Garcia, Periodic orbits and invariant cones in three-dimensional, piecewise linear systems, Discrete Contin. Dynam. Systems 35 (1) (2015) 59-72.

[23] Y. Iwatani, S. Hara, Stability tests and stabilization for piecewise linear systems based on poles and zeros of subsystems, Automatica 42 (2006) 1685-1695.

[24] M.K. Camlıbel, W.P.M.H. Heemels, J.M. Schumacher, Stability and controllability of planar bimodal linear complementarity systems, in: Proceedings of the 42nd IEEE Conference on Decision and Control, WeA05-1, Hawaii USA, December 2003, pp. 1651-1656. 